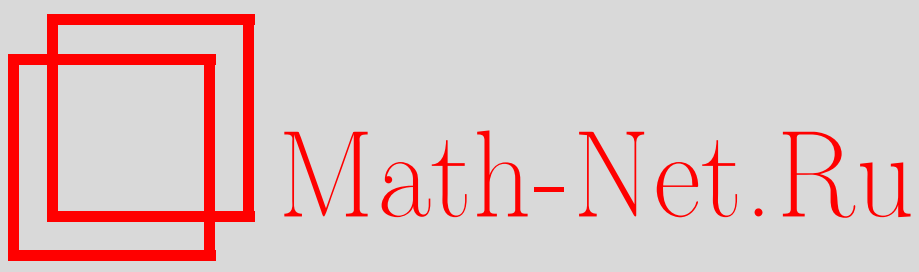

А. В. Боровских, Групповая классификация уравнений эйконала для трехмерной неоднородной среды, Матем. сб., 2004, том 195, номер 4, 23-64

DOI: https://doi.org/10.4213/sm813

Использование Общероссийского математического портала Math-Net.Ru подразумевает, что вы прочитали и согласны с пользовательским соглашением

http: //www.mathnet.ru/rus/agreement

Параметры загрузки:

IP: 52.6 .47 .48

26 апреля 2023 г., 10:18:49 
УДК 517.958

\section{А.В. Боровских \\ Групповая классификация уравнений эйконала для трехмерной неоднородной среды}

В настоящей работе изучается уравнение $(\nabla \psi)^{2}=1 / v^{2}(x, y, z)$, называемое уравнением эйконала. Это - уравнение характеристик для волновых уравнений в неоднородной среде, играющее ведущую роль при описании геометрии лучей и фронтов волн. В работе осуществляется полная групповая классификация семейства уравнений эйконала (уравнение определяется функцией $v(x, y, z)$, имеющей смысл скорости распространения возмущения в среде). В случаях уравнения с линейной и квадратичной функцией скорости $v(x, y, z)$ приводятся явные решения - эйконалы точечного источника - и полностью описывается геометрия лучей.

Библиографиия: 16 названий.

§1. Постановка проблемы, понятия, формулировки результатов, схема доказательства

1.1. Уравнение эйконала. Уравнение эйконала

$$
\left(\frac{\partial \psi}{\partial x}\right)^{2}+\left(\frac{\partial \psi}{\partial y}\right)^{2}+\left(\frac{\partial \psi}{\partial z}\right)^{2}=\frac{1}{v^{2}(x, y, z)}
$$

где $\psi$ - неизвестная функция, $v(x, y, z)$ - заданная функция, является уравнением характеристик для волновых уравнений различной природы: как акустических (cм. [1]-[6])

$$
m(x, y, z) \frac{\partial^{2} u}{\partial t^{2}}=\operatorname{div}(p(x, y, z) \operatorname{grad} u), \quad \frac{1}{v^{2}(x, y, z)} \frac{\partial^{2} u}{\partial t^{2}}=\Delta u,
$$

так и системы уравнений Максвелла (см., например, [4; гл. I, §5]).

Основное значение уравнения (1) состоит в том, что его решения позволяют описать фронты волн, распространяющихся в неоднородной среде, в виде соотношения $t=\psi(x, y, z)$ (более общие постановки, в которых $v$ зависит еше и от времени, см. в [7]). Язык лучей и фронтов, восходящий к древнегреческим представлениям об образе ( $\iota \kappa \kappa \widetilde{\omega} \nu$ - отсюда и термин “эйконал”) тела, отделяющемся от него и достигаюшем нашего глаза, до сих пор является самым эффективным в волновой теории

Работа выполнена при финансовой поддержке Госкомвуза Р $\Phi$ (грант № Е02-1.0-46), Российского фонда фундаментальных исследований (гранты № № 01-01-00417, 01-01-00418, 02-01-00307), программы "Университеты России" (грант № УР.04.01.004) и Программы поддержки ведущих научных школ РФ (грант № НШ-1643.2003.1). 
и употребляется повсеместно [1]-[9]. Однако само уравнение (1), за исключением случая $v(x, y, z)=$ const, исследовано весьма мало. Например, в [5] (см. также [10; гл. 3. $\S 2$, пा. 148,149$])$ решения соответствуюшего уравнения (1) предполагаются уже заданными. В [4] используются только первые члены асимптотических разложений решений (1), определяемые по сушеству из эталонной задачи о дифракции плоской волны на шаре. Некоторые частные случаи уравнения (1) исследованы в [2], [3] и особенно в [6], однако все полученные результаты либо связаны с предположением об изначальной разделяемости переменных, либо предполагают, что получаемые в процессе решения квадратуры уже известны. Анализ геометрии лучей в [8], [9], [11] с точки зрения теории особенностей отличается глубиной и обшностью, однако носит все-таки только качественный характер.

Такое "недостаточное" внимание к уравнению (1) нельзя считать случайным. Ввиду чрезвычайной обшности этого уравнения для его содержательного анализа необходимо иметь достаточный запас “явно решаемых" случаев для того, чтобы теми или иными интегральными соображениями получать выводы и для случаев "нерешаемых". По существу же до сих пор никаких "явно решаемых" случаев кроме случая постоянной $v(x, y, z)$ известно не было.

Групповая классификация как раз помогает препарировать семейство уравнений с целью выделения из них наиболее доступных для анализа. По существу эта доступность измеряется размерностью той группы преобразований, которая оставляет уравнение инвариантным.

В заключение отметим, что для трех полученных нами "главных" случаев, когда группа, оставляющая уравнение (1) инвариантным, имеет максимальную размерность (а именно 15) - случаев постоянной, линейной и квадратичной $v(x, y, z)$, уравнение (1), как оказалось, явно решается: удается найти эйконал точечного источника (он оказывается подвижной сферой) и как следствие полностью описать геометрию лучей. Эти результаты, подтверждающие эффективность группового анализа, приводятся нами в последнем параграфе.

1.2. Группы симметрий. Напомним (подробное изложение концепций и методов группового анализа см. в [12]-[14]), что точечное преобразование пространства переменных (в нашем случае - $(x, y, z, \psi))$ называется симметрией дифференциального уравнения, если оно оставляет это уравнение инвариантным (смысл понятия инвариантности мы уточним в п. 2.1). Множество симметрий конкретного уравнения имеет структуру группы (относительно операции суперпозиции), непрерывная составляюшая этой группы обычно параметризуется одним или несколькими параметрами, приобретая тем самым и структуру многообразия и становясь группой Ли (так называют многообразие, на котором определены групповые операции). Это позволяет определять размерность группы симметрий просто как размерность касательного пространства (она одна и та же во всех точках: в силу группового свойства окрестность каждой точки изоморфна окрестности тождественного преобразования).

С конечномерной группой Ли точечных преобразований пространства переменных $(x, y, z, \psi)$ связана алгебра векторных полей, порождающих одномерные подгруппы этой группы (алгебра Ли). Компоненты такого векторного поля мы будем обозначать через $\xi, \eta, \zeta, \varphi$ соответственно. Они являются функциями от четырех переменных $x, y, z, \psi$, и однопараметрическая подгруппа преобразований, порож- 
даемая таким векторным полем, вычисляется как решение системы $\dot{x}=\xi, \dot{y}=\eta$, $\dot{z}=\zeta, \dot{\psi}=\varphi$, где точка означает дифференцирование относительно параметризации этой подгруппы.

Однопараметрическая группа точечных преобразований пространства $\mathbb{R}^{4}$ задает однопараметрическую группу преобразований в множестве функций, заданных на этом пространстве, инфинитезимальная образующая которой оказывается дифференциальным оператором

$$
\Xi=\xi \partial_{x}+\eta \partial_{y}+\zeta \partial_{z}+\varphi \partial_{\psi}
$$

$\left(\partial_{\alpha}-\right.$ дифференцирования по соответствующим переменным $)$, поэтому алгебру Ли исходной группы обычно отождествляют с алгеброй операторов вида (2).

Преимушество языка алгебр Ли состоит в том, что группа однозначно определяется своей алгеброй Ли, а последняя имеет структуру линейного пространства. Поэтому описать группу - это значит предъявить конкретные выражения $\xi(x, y, z, \psi), \eta(x, y, z, \psi), \zeta(x, y, z, \psi), \varphi(x, y, z, \psi)$ коэффициентов в $(2)$, в которых фигурируют произвольные константы, входящие в выражения как коэффищиенты линейной комбинации. Количество констант (естественно, с оговоркой о линейной независимости соответствующих векторных полей) и является размерностью группы. Если константы не совсем произвольные, а связаны некоторыпи линейными соотношениями (нам будет удобно использовать и такой вариант), то размерность группы, естественно, - это количество констант минус количество соотношений.

Для сокращения записи нам будет удобно ввести обозначения для некоторых типичных для нашего уравнения подгрупп (а точнее, соответствующих им подалгебр):

$$
\Xi_{\uparrow}(H, I, J)=H \partial_{x}+I \partial_{y}+J \partial_{z}
$$

порождает подгруппу сдвигов (при фиксированных $H, I, J$ - вдоль $(H, I, J))$;

$$
\Xi_{\circ}(E, F, G)=(G y-F z) \partial_{x}+(-G x+E z) \partial_{y}+(F x-E y) \partial_{z}
$$

порождает подгруппу врашений (при фиксированных $E, F, G$ - вокруг $(E, F, G)$ );

$$
\Xi_{*}=x \partial_{x}+y \partial_{y}+z \partial_{z}
$$

порождает подгруппу растяжений в пространстве переменных $(x, y, z)$;

$$
\begin{aligned}
\Xi_{\infty}(A, B, C, k)= & \left(A\left(x^{2}-y^{2}-z^{2}-k\right)+2 B x y+2 C x z\right) \partial_{x} \\
& +\left(B\left(y^{2}-x^{2}-z^{2}-k\right)+2 A x y+2 C y z\right) \partial_{y} \\
& +\left(C\left(z^{2}-x^{2}-y^{2}-k\right)+2 A x z+2 B y z\right) \partial_{z}
\end{aligned}
$$

порождает подгруппу циклонообразных движений (при фиксированных $A, B, C-$ с осью $l=(A, B, C)$ ), эти движения осесимметричны, их плоские сечения изображены на рис. 1 для $k=\nu^{2}, k=-\nu^{2}$ и на рис. 2 - для $k=0$.

Алгебра $\Xi_{\infty}(A, B, C, 0)+D \Xi_{*}+\Xi_{\circ}(E, F, G)+\Xi_{\uparrow}(H, I, J)$ с произвольными постоянными (не зависящими от $\psi$ ) коэффициентами - это алгебра группы конформных преобразований пространства переменных $(x, y, z)[15 ; \S 9]$, однако мы будем 

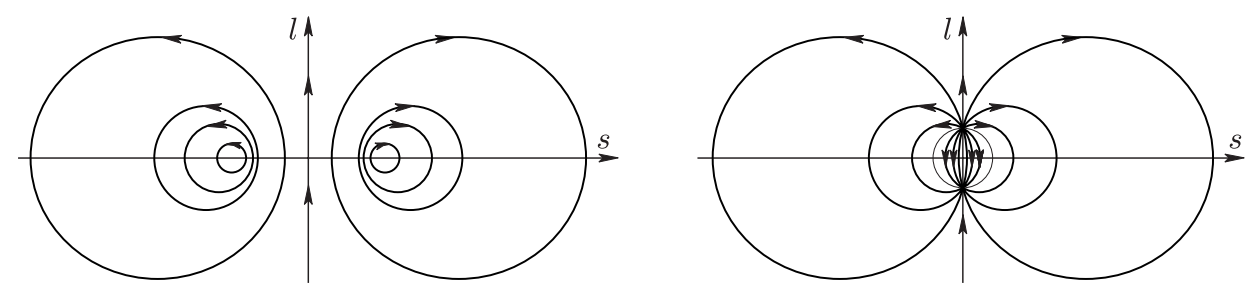

Рис. 1. Плоские фазовые портреты: слева $k=\nu^{2}$, справа $k=-\nu^{2}$

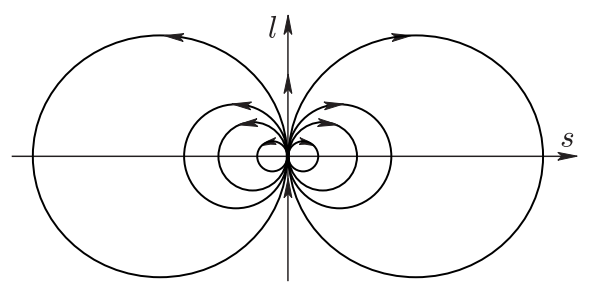

Рис. 2. Плоский фазовый портрет в случае $k=0$

использовать $\Xi_{\alpha}$ не столько как подалгебры, отвечаюшие подгруппам конформной группы, сколько как "базу" для построения компонент более сложных алгебр, в которых параметры зависят еще и от $\psi$. Например,

$$
\begin{aligned}
\left(D_{1} \psi+D_{2}\right) \Xi_{*}=\left(D_{1} \psi+D_{2}\right) x \partial_{x}+\left(D_{1} \psi+D_{2}\right) y \partial_{y}+\left(D_{1} \psi+D_{2}\right) z \partial_{z}, \\
\Xi_{\infty}\left(A, B, C,-\psi^{2} v^{2}\right)=\left(A\left(\psi^{2} v^{2}+x^{2}-y^{2}-z^{2}\right)+2 B x y+2 C x z\right) \partial_{x} \\
\quad+\left(B\left(\psi^{2} v^{2}+y^{2}-x^{2}-z^{2}\right)+2 A x y+2 C y z\right) \partial_{y} \\
\quad+\left(C\left(\psi^{2} v^{2}+z^{2}-x^{2}-y^{2}\right)+2 A x z+2 B y z\right) \partial_{z}, \\
\Xi_{\circ}\left(\mathrm{Ch}_{w}\left(E_{1}, E_{2}\right), \mathrm{Ch}_{w}\left(F_{1}, F_{2}\right), \mathrm{Ch}_{w}\left(G_{1}, G_{2}\right)\right) \\
=\left(\left(G_{1} \operatorname{ch}(w \psi)+G_{2} \operatorname{sh}(w \psi)\right) y-\left(F_{1} \operatorname{ch}(w \psi)+F_{2} \operatorname{sh}(w \psi)\right) z\right) \partial_{x} \\
\quad+\left(-\left(G_{1} \operatorname{ch}(w \psi)+G_{2} \operatorname{sh}(w \psi)\right) x+\left(E_{1} \operatorname{ch}(w \psi)+E_{2} \operatorname{sh}(w \psi)\right) z\right) \partial_{y} \\
\quad+\left(\left(F_{1} \operatorname{ch}(w \psi)+F_{2} \operatorname{sh}(w \psi)\right) x-\left(E_{1} \operatorname{ch}(w \psi)+E_{2} \operatorname{sh}(w \psi)\right) y\right) \partial_{z} .
\end{aligned}
$$

В последнем равенстве употреблено одно из серии используемых далее обозначений для тригонометрических и гиперболических функциональных зависимостей от $\psi$ с коэффициентами $\lambda_{i}$ (которые обычно произвольные) и с параметром $w$ (который обычно фиксирован и определен параметрами функции $v(x, y, z))$ :

$$
\begin{aligned}
\operatorname{Cos}_{w}\left(\lambda_{1}, \lambda_{2}\right) & =\lambda_{1} \cos (w \psi)+\lambda_{2} \sin (w \psi), \\
\operatorname{Sin}_{w}\left(\lambda_{1}, \lambda_{2}\right) & =-\lambda_{1} \sin (w \psi)+\lambda_{2} \cos (w \psi), \\
\operatorname{Ch}_{w}\left(\lambda_{1}, \lambda_{2}\right) & =\lambda_{1} \operatorname{ch}(w \psi)+\lambda_{2} \operatorname{sh}(w \psi), \\
\operatorname{Sh}_{w}\left(\lambda_{1}, \lambda_{2}\right) & =\lambda_{1} \operatorname{sh}(w \psi)+\lambda_{2} \operatorname{ch}(w \psi) .
\end{aligned}
$$




\section{3. Групповая классификация уравнения (1).}

Теорема 1. І. Группа симметрий уравнения эйконала (1) является 15-мерной для следующих функиий скорости $v(x, y, z)$ : постоянной

$$
v(x, y, z) \equiv \mathrm{const},
$$

линейной

$$
v(x, y, z)=P x+Q y+R z, \quad P^{2}+Q^{2}+R^{2}>0,
$$

и квадратичной одного из трех типов

$$
\begin{array}{ll}
v(x, y, z)=w \cdot\left(x^{2}+y^{2}+z^{2}-\nu^{2}\right), & \nu>0 \\
v(x, y, z)=w \cdot\left(x^{2}+y^{2}+z^{2}+\nu^{2}\right), & \nu>0 \\
v(x, y, z)=w \cdot\left(x^{2}+y^{2}+z^{2}\right) . &
\end{array}
$$

Соответствующие алгебры Ли перечислены в таблице 1.

II. Группа симметрий уравнения әйконала является 4 -мерной $\partial л я ~ v(x, y, z)$, принадлежащей одному из четырех семейств функций $(V(\cdot)-$ произвольная функция): плоских

$$
v(x, y, z)=V(P x+Q y+R z), \quad P^{2}+Q^{2}+R^{2}=1,
$$

сферических

$$
v(x, y, z)=V\left(x^{2}+y^{2}+z^{2}\right),
$$

чилиндрических

$$
v(x, y, z)=(-G x+E z) V\left(\frac{G y-F z}{-G x+E z}\right), \quad G \neq 0, \quad E^{2}+F^{2}+G^{2}=1,
$$

и осесимметричных

$$
v(x, y, z)=\left(x^{2}+y^{2}+z^{2} \pm \nu^{2}\right) V\left(\frac{P x+Q y+R z}{x^{2}+y^{2}+z^{2} \pm \nu^{2}}\right), \quad P^{2}+Q^{2}+R^{2}=1 .
$$

Соответствующие алгебры Ли перечислены в табличе 2.

Каждое из указанных семейств содержит по нескольку (четыре, шесть, шесть и шестнадцать соответственно) конечномерных подсемейств, для которых группы симметрий уравнения (1) шестимерны; кроме того, семейство (II.1) имеет одно подсемейство, для которого группа симметрий уравнения (1) пятимерна. Списки этих подсемейств для каждого семейства и соответствующие алгебры Ли приведены в таблицах 3-7.

III. Для 11 семейств функиий скорости $v(x, y, z)$, описываемых произвольными функциями от двух аргументов (см. таблицы 8 и 9), группа симметрий уравнения (1) двумерна.

IV. Во всех остальных случаях группа симметрий уравнения (1) одномерна (әто - группа сдвигов по $\psi$ с алгеброй $L \partial_{\psi}$ ). 
Всюду в формулах (I.1)-(I.5), (II.1)-(II.4) и в таблицах 1-9 предполагается, что функщия $v(x, y, z)$ приведена к указанному виду с помощью подходящего выбора начала координат, а для семейств раздела III - еще и за счет подходящего ортогонального преобразования системь координат (новье координать обозначаются через $(l, s, p))$.

ТАБЛицА 1. Алгебры Ли групп симметрий уравнения (1) с функциями скорости (I.1)-(I.5)

\begin{tabular}{|c|c|}
\hline $\begin{array}{c}v(x, y, z) \\
\text { константы } \\
\text { условия }\end{array}$ & Алгебра Ли $\Xi$ \\
\hline $\begin{array}{c}v \text { (三 const) } \\
A, B, C, D_{i}, E, F, G, \\
H_{i}, I_{i}, J_{i}, L\end{array}$ & $\begin{array}{l}\left(\Xi_{\infty}\left(A, B, C,-\psi^{2} v^{2}\right)+2(A x+B y+C z) \psi \partial_{\psi}\right) \\
+D_{1}\left(2 \psi\left(x \partial_{x}+y \partial_{y}+z \partial_{z}\right)+\frac{\psi^{2} v^{2}+x^{2}+y^{2}+z^{2}}{v^{2}} \partial_{\psi}\right) \\
+\left(\psi\left(H_{1} \partial_{x}+I_{1} \partial_{y}+J_{1} \partial_{z}\right)+\frac{1}{v^{2}}\left(H_{1} x+I_{1} y+J_{1} z\right) \partial_{\psi}\right) \\
+D_{2}\left(\Xi_{*}+\psi \partial_{\psi}\right)+\Xi_{\circ}(E, F, G)+\Xi_{\uparrow}\left(H_{2}, I_{2}, J_{2}\right)+L \partial_{\psi}\end{array}$ \\
\hline $\begin{array}{c}P x+Q y+R z, \\
P^{2}+Q^{2}+R^{2}=w^{2}, \\
w>0 \\
\lambda_{i}, E_{i}, F_{i}, G_{i}, \varkappa_{i}, \\
A, B, C, D, \theta, H, I, J, L, \\
P E_{i}+Q F_{i}+R G_{i}=0, \\
P A+Q B+R C=0, \\
P H+Q I+R J=0\end{array}$ & $\begin{array}{l}\left(\mathrm{Ch}_{w}\left(\lambda_{1}, \lambda_{2}\right) \Xi_{\infty}(P, Q, R, 0)+\mathrm{Sh}_{w}\left(\lambda_{1}, \lambda_{2}\right) \frac{w\left(x^{2}+y^{2}+z^{2}\right)}{P x+Q y+R z} \partial_{\psi}\right) \\
+\left(\Xi_{\circ}\left(\mathrm{Ch}_{w}\left(E_{1}, E_{2}\right), \mathrm{Ch}_{w}\left(F_{1}, F_{2}\right), \mathrm{Ch}_{w}\left(G_{1}, G_{2}\right)\right)\right. \\
+\frac{1}{w(P x+Q y+R z)}\left(\mathrm{Sh}_{w}\left(E_{1}, E_{2}\right)(R y-Q z)\right. \\
\left.\left.+\mathrm{Sh}_{w}\left(F_{1}, F_{2}\right)(P z-R x)+\mathrm{Sh}_{w}\left(G_{1}, G_{2}\right)(Q x-P y)\right) \partial_{\psi}\right) \\
+\left(\mathrm{Ch}_{w}\left(\varkappa_{1}, \varkappa_{2}\right) \Xi_{\uparrow}(P, Q, R)-\mathrm{Sh}_{w}\left(\varkappa_{1}, \varkappa_{2}\right) \frac{w}{P x+Q y+R z} \partial_{\psi}\right) \\
+\Xi_{\infty}(A, B, C, 0)+D \Xi_{*}+\theta \Xi_{\circ}(P, Q, R) \\
+\Xi_{\uparrow}(H, I, J)+L \partial_{\psi}\end{array}$ \\
\hline $\begin{array}{c}w\left(x^{2}+y^{2}+z^{2}-\nu^{2}\right), \\
w \neq 0, \quad \nu \neq 0 \\
A_{i}, B_{i}, C_{i}, D_{i} \\
E, F, G, H, I, J, L\end{array}$ & $\begin{aligned}\left(\Xi_{\infty}\left(\mathrm{Ch}_{2 w \nu}\left(A_{1}, A_{2}\right), \mathrm{Ch}_{2 w \nu}\left(B_{1}, B_{2}\right), \mathrm{Ch}_{2 w \nu}\left(C_{1}, C_{2}\right),-\nu^{2}\right)\right. \\
\quad-\left(\mathrm{Sh}_{2 w \nu}\left(A_{1}, A_{2}\right) x+\mathrm{Sh}_{2 w \nu}\left(B_{1}, B_{2}\right) y\right. \\
\left.\left.\quad+\mathrm{Sh}_{2 w \nu}\left(C_{1}, C_{2}\right) z\right) \frac{2 \nu}{w\left(x^{2}+y^{2}+z^{2}-\nu^{2}\right)} \partial_{\psi}\right) \\
\quad+\left(\mathrm{Ch}_{2 w \nu}\left(D_{1}, D_{2}\right) \Xi_{*}\right. \\
\left.\quad-\mathrm{Sh}_{2 w \nu}\left(D_{1}, D_{2}\right) \frac{x^{2}+y^{2}+z^{2}+\nu^{2}}{2 w \nu\left(x^{2}+y^{2}+z^{2}-\nu^{2}\right)} \partial_{\psi}\right) \\
\quad+\Xi_{\circ}(E, F, G)-\frac{1}{2 \nu^{2}} \Xi_{\infty}\left(H, I, J, \nu^{2}\right)+L \partial_{\psi}\end{aligned}$ \\
\hline
\end{tabular}


ТАБлИцА 1 (продолжение)

\begin{tabular}{|c|c|}
\hline $\begin{array}{c}v(x, y, z) \\
\text { константы } \\
\text { условия }\end{array}$ & Алгебра Ли $\Xi$ \\
\hline $\begin{array}{c}w\left(x^{2}+y^{2}+z^{2}+\nu^{2}\right) \\
w>0, \quad \nu \neq 0 \\
A_{i}, B_{i}, C_{i}, D_{i} \\
E, F, G, H, I, J, L\end{array}$ & $\begin{aligned}\left(\Xi_{\infty}\left(\operatorname{Cos}_{2 w \nu}\left(A_{1}, A_{2}\right), \operatorname{Cos}_{2 w \nu}\left(B_{1}, B_{2}\right), \operatorname{Cos}_{2 w \nu}\left(C_{1}, C_{2}\right), \nu^{2}\right)\right. \\
\quad-\left(\operatorname{Sin}_{2 w \nu}\left(A_{1}, A_{2}\right) x+\operatorname{Sin}_{2 w \nu}\left(B_{1}, B_{2}\right) y\right. \\
\left.\left.\quad+\operatorname{Sin}_{2 w \nu}\left(C_{1}, C_{2}\right) z\right) \frac{2 \nu}{w\left(x^{2}+y^{2}+z^{2}+\nu^{2}\right)} \partial_{\psi}\right) \\
\quad+\left(\operatorname{Cos}_{2 w \nu}\left(D_{1}, D_{2}\right) \Xi_{*}\right. \\
\left.\quad+\operatorname{Sin}_{2 w \nu}\left(D_{1}, D_{2}\right) \frac{x^{2}+y^{2}+z^{2}-\nu^{2}}{2 w \nu\left(x^{2}+y^{2}+z^{2}+\nu^{2}\right)} \partial_{\psi}\right) \\
\quad+\Xi_{\circ}(E, F, G)+\frac{1}{2 \nu^{2}} \Xi_{\infty}\left(H, I, J,-\nu^{2}\right)+L \partial_{\psi}\end{aligned}$ \\
\hline $\begin{array}{c}w\left(x^{2}+y^{2}+z^{2}\right), \\
w>0 \\
A_{i}, B_{i}, C_{i}, D_{i}, \\
E, F, G, H, I, J, L\end{array}$ & $\begin{array}{l}\left(\Xi_{\infty}\left(A_{1} \psi+A_{2}, B_{1} \psi+B_{2}, C_{1} \psi+C_{2}, 0\right)-\frac{A_{1} x+B_{1} y+C_{1} z}{w^{2}\left(x^{2}+y^{2}+z^{2}\right)} \partial_{\psi}\right) \\
+\left(\Xi_{\infty}\left(w^{2} \psi^{2} H, w^{2} \psi^{2} I, w^{2} \psi^{2} J,-1\right)-2 \psi \frac{H x+I y+J z}{x^{2}+y^{2}+z^{2}} \partial_{\psi}\right) \\
+D_{1}\left(2 \psi\left(x \partial_{x}+y \partial_{y}+z \partial_{z}\right)-\left(\psi^{2}+\frac{1}{w^{2}\left(x^{2}+y^{2}+z^{2}\right)}\right) \partial_{\psi}\right) \\
+D_{2}\left(\Xi_{*}-\psi \partial_{\psi}\right)+\Xi_{\circ}(E, F, G)+L \partial_{\psi}\end{array}$ \\
\hline
\end{tabular}

1.4. Схема доказательства. Опишем вкратце схему доказательства, приведенного в последующих параграфах. Первый шаг - это получение определяющих уравнений, т.е. дифференциальных уравнений, связывающих $\xi, \eta, \zeta, \varphi$ и $v(x, y, z)$ и являюшихся необходимыми и достаточньми условиями инвариантности уравнения эйконала с функцией $v(x, y, z)$ относительно соответствующей групшы преобразований. Второй этап - это решение части из этих уравнений, позволяющее описать общий вид функций $\xi, \eta, \zeta$ как функций от $(x, y, z)$.

Третий этап - это “грубая" классификация с выделением семейств (II.1)-(II.4). Дело в том, что в определяющих уравнениях условия относительно $\varphi$ выглядят просто как задание градиента $\varphi$ по переменным $(x, y, z)$, причем правые части этих равенств выражены некоторым образом через $\xi, \eta, \zeta$. Для того чтобы эти правые части действительно были компонентами градиента, необходимо выполнение дополнительных условий (условия полного дифференциала), что налагает дополнительные ограничения на $\xi, \eta, \zeta$. Эти ограничения и позволяют произвести первичную сепарацию.

Следует отметить, что коэффициенты, фигурирующие в описании семейств (II.1)-(II.4) при грубой классификации, получаются, вообше говоря, зависящими от $\psi$. А поскольку функция $v$ от $\psi$ зависеть не должна (просто по постановке задачи - неавтономньй случай уравнения (1) мы не рассматриваем), приходится 
ТАБЛицА 2. Алгебры Ли групп симметрий уравнения (1) с функциями скорости из семейств (II.1)-(II.4); $V(\cdot)$ - произвольная функция

\begin{tabular}{|c|c|}
\hline $\begin{array}{c}v(x, y, z) \\
\text { константы } \\
\text { условия }\end{array}$ & Алгебра Ли $\Xi$ \\
\hline$\frac{V(P x+Q y+R z), \quad P^{2}+Q^{2}+R^{2}=1}{\Delta, H, I, J, L, \quad P H+Q I+R J=0}$ & $\begin{aligned} \Xi_{\mathrm{II} .1}=\Delta & \Delta \Xi_{\circ}(P, Q, R) \\
& +\Xi_{\uparrow}(H, I, J)+L \partial_{\psi}\end{aligned}$ \\
\hline$\frac{V\left(x^{2}+y^{2}+z^{2}\right)}{E, F, G, L}$ & $\Xi_{\mathrm{II} .2}=\Xi_{\circ}(E, F, G)+L \partial_{\psi}$ \\
\hline $\begin{array}{c}(-G x+E z) V\left(\frac{G y-F z}{-G x+E z}\right) \\
E^{2}+F^{2}+G^{2}=1, \quad G \neq 0 \\
\Delta, D, \varkappa, L\end{array}$ & $\begin{aligned} \Xi_{\mathrm{II} .3}=\Delta \Xi_{\infty}(E, F, G, 0)+D \Xi_{*} \\
+\varkappa \Xi_{\uparrow}(E, F, G)+L \partial_{\psi}\end{aligned}$ \\
\hline $\begin{array}{c}\left(x^{2}+y^{2}+z^{2} \pm \nu^{2}\right) V\left(\frac{P x+Q y+R z}{x^{2}+y^{2}+z^{2} \pm \nu^{2}}\right. \\
\frac{P^{2}+Q^{2}+R^{2}=1}{A, B, C, \theta, L, \quad P A+Q B+R C=0}\end{array}$ & $\begin{aligned} \Xi_{\mathrm{II} .4}=\Xi_{\infty}\left(A, B, C, \mp \nu^{2}\right) \\
+\theta \Xi_{\circ}(P, Q, R)+L \partial_{\psi}\end{aligned}$ \\
\hline
\end{tabular}

ТАБЛИцА 3. Подсемейства семейства (II.1): $v(x, y, z)=w V(\chi)$, где $w \neq 0, \chi=P x+Q y+R z$ (здесь $\left.P^{2}+Q^{2}+R^{2}=1\right)$, вид функции $V(\cdot)$ указан в таблице; всюду $k \neq 0, h=$ const, произвольные постоянные $\theta_{i}, \Delta, H, I, J, L$ удовлетворяют условию $P H+Q I+R J=0$

\begin{tabular}{|c|c|}
\hline$V(\chi)$ & Алгебра Ли $\Xi$ \\
\hline$\chi^{k+1}$ & $\Xi_{\mathrm{II} .1}+\theta_{1}\left(\Xi_{*}-k \psi \partial_{\psi}\right)$ \\
\hline$e^{k \chi}$ & $\Xi_{\mathrm{II} .1}+\theta_{1}\left(2 \psi \Xi_{\uparrow}(P, Q, R)-\left(k \psi^{2}+\frac{1}{w^{2} k} e^{-2 k \chi}\right) \partial_{\psi}\right)$ \\
$+\theta_{2}\left(\Xi_{\uparrow}(P, Q, R)-k \psi \partial_{\psi}\right)$
\end{tabular}


ТАБлицА 4. Подсемейства семейства (II.2): $v(x, y, z)=w \sqrt{x^{2}+y^{2}+z^{2}} V(\chi)$, где $w \neq 0, \quad \chi=\frac{1}{2} \ln \left(x^{2}+y^{2}+z^{2}\right)$, вид функции $V(\cdot)$ указан в таблице; всюду $k \neq 0, h=$ const, произвольные константы $-D_{i}, E, F, G, L$

\begin{tabular}{|c|c|}
\hline$V(\chi)$ & Алгебра Ли $\Xi$ \\
\hline 1 & $\Xi_{\mathrm{II} .2}+\left(D_{1} \psi+D_{2}\right) \Xi_{*}+D_{1} \frac{1}{w^{2}} \chi \partial_{\psi}$ \\
\hline$e^{k \chi}$ & $\Xi_{\mathrm{II} .2}+\left(2 D_{1} \psi+D_{2}\right) \Xi_{*}-\left(D_{1}\left(\frac{1}{k w^{2}} e^{-2 k \chi}+k \psi^{2}\right)+D_{2} k \psi\right) \partial_{\psi}$ \\
\hline $\cos (k \chi+h)$ & $\Xi_{\mathrm{II} .2}+\mathrm{Ch}_{k w}\left(D_{1}, D_{2}\right) \Xi_{*}+\frac{1}{w} \operatorname{Sh}_{k w}\left(D_{1}, D_{2}\right) \operatorname{tg}(k \chi+h) \partial_{\psi}$ \\
\hline $\operatorname{sh}(k \chi+h)$ & $\Xi_{\mathrm{II} .2}+\mathrm{Ch}_{k w}\left(D_{1}, D_{2}\right) \Xi_{*}-\frac{1}{w} \operatorname{Sh}_{k w}\left(D_{1}, D_{2}\right) \operatorname{cth}(k \chi+h) \partial_{\psi}$ \\
\hline $\operatorname{ch}(k \chi+h)$ & $\Xi_{\mathrm{II} .2}+\operatorname{Cos}_{k w}\left(D_{1}, D_{2}\right) \Xi_{*}-\frac{1}{w} \operatorname{Sin}_{k w}\left(D_{1}, D_{2}\right) \operatorname{th}(k \chi+h) \partial_{\psi}$ \\
\hline$\chi+h$ & $\Xi_{\mathrm{II} .2}+\mathrm{Ch}_{w}\left(D_{1}, D_{2}\right) \Xi_{*}-\operatorname{Sh}_{w}\left(D_{1}, D_{2}\right) \frac{1}{w(\chi+h)} \partial_{\psi}$ \\
\hline
\end{tabular}

ТАБЛИцА 5. Подсемейства семейства (II.3):

$v(x, y, z)=w \sqrt{(G y-F z)^{2}+(-G x+E z)^{2}+(F x-E y)^{2}} V(\chi)$,

где $w \neq 0, E^{2}+F^{2}+G^{2}=1, \chi=\operatorname{arctg}\left(\frac{G(G y-F z)+E(E y-F x)}{-G x+E z}\right)$, вид функции $V(\cdot)$ указан в таблице; всюду $k \neq 0, h=$ const, произвольные константы - $D, \Delta, \theta_{i}, \varkappa, L$

\begin{tabular}{|c|c|}
\hline$V(\chi)$ & Алгебра Ли $\Xi$ \\
\hline 1 & $\Xi_{\mathrm{II} .3}+\left(\theta_{1} \psi+\theta_{2}\right) \Xi_{\circ}(E, F, G)+\frac{\theta_{1}}{w^{2}} \chi \partial_{\psi}$ \\
\hline$e^{k \chi}$ & $\Xi_{\mathrm{II} .3}+\left(2 \theta_{1} \psi+\theta_{2}\right) \Xi_{\circ}(E, F, G)-\left(\frac{\theta_{1}}{k w^{2}} e^{-2 k \chi}+k \theta_{1} \psi^{2}+k \theta_{2} \psi\right) \partial_{\psi}$ \\
\hline $\cos (k \chi+h)$ & $\Xi_{\mathrm{II} .3}+\mathrm{Ch}_{k w}\left(\theta_{1}, \theta_{2}\right) \Xi_{\circ}(E, F, G)+\frac{1}{w} \operatorname{Sh}_{k w}\left(\theta_{1}, \theta_{2}\right) \operatorname{tg}(k \chi+h) \partial_{\psi}$ \\
\hline $\operatorname{sh}(k \chi+h)$ & $\Xi_{\mathrm{II} .3}+\mathrm{Ch}_{k w}\left(\theta_{1}, \theta_{2}\right) \Xi_{\circ}(E, F, G)-\frac{1}{w} \operatorname{Sh}_{k w}\left(\theta_{1}, \theta_{2}\right) \operatorname{cth}(k \chi+h) \partial_{\psi}$ \\
\hline $\operatorname{ch}(k \chi+h)$ & $\Xi_{\mathrm{II} .3}+\operatorname{Cos}_{k w}\left(\theta_{1}, \theta_{2}\right) \Xi_{\circ}(E, F, G)-\frac{1}{w} \operatorname{Sin}_{k w}\left(\theta_{1}, \theta_{2}\right) \operatorname{th}(k \chi+h) \partial_{\psi}$ \\
\hline$\chi+h$ & $\Xi_{\mathrm{II} .3+\mathrm{Ch}_{k w}\left(\theta_{1}, \theta_{2}\right) \Xi_{\circ}(E, F, G)-\operatorname{Sh}_{w}\left(\theta_{1}, \theta_{2}\right) \frac{G}{w(\chi+h)} \partial_{\psi}}$ \\
\hline
\end{tabular}


ТАБЛИцА 6. Подсемейства семейства (II.4):

$v(x, y, z)=w \sqrt{\left(x^{2}+y^{2}+z^{2} \pm \nu^{2}\right)^{2} \mp 4 \nu^{2}(P x+Q y+R z)^{2}} V(\chi)$, где $w \neq 0, P^{2}+Q^{2}+R^{2}=1$, вид функции $V(\cdot)$ указан в таблице, а выражения $\chi$, соответствующие различньм случаям значений $\pm \nu^{2}$ (т.е. $+\nu^{2},-\nu^{2}$ или 0 ), приведены в таблице $7 ;$ всюду $k \neq 0$, произволњные константы $\Delta_{1}, \Delta_{2}, A, B, C, \theta, L$ удовлетворяют условию $P A+Q B+R C=0$

\begin{tabular}{|c|c|}
\hline$V(\chi)$ & Алгебра Ли $\Xi$ \\
\hline 1 & $\Xi_{\mathrm{II} .4}-\left(\Delta_{1} \psi+\Delta_{2}\right) \Xi_{\infty}\left(P, Q, R, \pm \nu^{2}\right)+\frac{\Delta_{1}}{w^{2}} \chi \partial_{\psi}$ \\
\hline$e^{k \chi}$ & $\Xi_{\mathrm{II} .4}-\left(2 \Delta_{1} \psi+\Delta_{2}\right) \Xi_{\infty}\left(P, Q, R, \pm \nu^{2}\right)$ \\
\hline $\cos (k \chi+h)$ & $-\left(\Delta_{1}\left(k \psi^{2}+\frac{e^{-2 k \chi}}{k w^{2}}\right)+\Delta_{2} k \psi\right) \partial_{\psi}$ \\
\hline $\operatorname{sh}(k \chi+h)$ & $\Xi_{\mathrm{II} .4}-\mathrm{Ch}_{w k}\left(\Delta_{1}, \Delta_{2}\right) \Xi_{\infty}\left(P, Q, R, \pm \nu^{2}\right)$ \\
& $+\frac{1}{w} \operatorname{Sh}_{w k}\left(\Delta_{1}, \Delta_{2}\right) \operatorname{tg}(k \chi+h) \partial_{\psi}$ \\
\hline $\operatorname{ch}(k \chi+h)$ & $\Xi_{\mathrm{II} .4}-\mathrm{Ch}_{w k}\left(\Delta_{1}, \Delta_{2}\right) \Xi_{\infty}\left(P, Q, R, \pm \nu^{2}\right)$ \\
& $-\frac{1}{w} \operatorname{Sh}_{w k}\left(\Delta_{1}, \Delta_{2}\right) \operatorname{cth}(k \chi+h) \partial_{\psi}$ \\
\hline$\chi+h$ & $\Xi_{\mathrm{II} .4}-\operatorname{Cos}_{w k}\left(\Delta_{1}, \Delta_{2}\right) \Xi_{\infty}\left(P, Q, R, \pm \nu^{2}\right)$ \\
$-\frac{1}{w} \operatorname{Sin}_{w k}\left(\Delta_{1}, \Delta_{2}\right) \operatorname{th}(k \chi+h) \partial_{\psi}$
\end{tabular}

ТАБлИцА 7. Вид функции скорости и выражения для $\chi(r)$, соответствующие различным случаям значений $\pm \nu^{2}$

\begin{tabular}{|c|c|}
\hline$v(x, y, z)$ & $\chi$ \\
\hline $\begin{array}{c}w\left(\left(x^{2}+y^{2}+z^{2}+\nu^{2}\right)^{2}\right. \\
\left.-4 \nu^{2}(P x+Q y+R z)^{2}\right)^{1 / 2} V(\chi)\end{array}$ & $\frac{1}{4 \nu} \ln \frac{(x+P \nu)^{2}+(y+Q \nu)^{2}+(z+R \nu)^{2}}{(x-P \nu)^{2}+(y-Q \nu)^{2}+(z-R \nu)^{2}}$ \\
\hline $\begin{array}{c}w\left(\left(x^{2}+y^{2}+z^{2}-\nu^{2}\right)^{2}\right. \\
\left.+4 \nu^{2}(P x+Q y+R z)^{2}\right)^{1 / 2} V(\chi)\end{array}$ & $\frac{1}{2 \nu} \operatorname{arctg}\left(2 \nu \frac{P x+Q y+R z}{x^{2}+y^{2}+z^{2}-\nu^{2}}\right)$ \\
\hline$w\left(x^{2}+y^{2}+z^{2}\right) V(\chi)$ & $\frac{P x+Q y+R z}{x^{2}+y^{2}+z^{2}}$ \\
\hline
\end{tabular}


ТАБлицА 8. Семейства из раздела III: $v=v(l, s, p)=w(l, s, p) \sqrt{r^{0}} V\left(r^{-} / r^{0}, r\right)$, где $(l, s, p)$ - выбранная подходящим образом ортогональная система координат, $w(l, s, p)$ - фиксированная функция, конкретньй вид которой указан в таблице, $r^{-} / r^{0}$ - рациональньй, а $r$ - трансцендентный интеграл системы $d l /\left(l^{2}-s^{2}-p^{2} \mp \nu^{2}\right)=d s /(2 l s+\theta p+\lambda)=d p /(2 l p-\theta s)$, конкретные выражения для $r^{0}, r^{-}$и в некоторых случаях для $r$ также приведены в таблице, там же указаны, где необходимо, выражения для $\theta, \lambda, \pm \nu^{2} ; V(\cdot, \cdot)-$ произвольная функция, группа симметрий во всех случаях имеет алгебру Ли $\Xi=\Delta\left(\left(l^{2}-s^{2}-p^{2} \mp \nu^{2}\right) \partial_{l}+(2 l s+\theta p+\lambda) \partial_{s}+(2 l p-\theta s) \partial_{p}+M \psi \partial_{\psi}\right)+L \partial_{\psi}$

с произвольньми константами $\Delta, L$, значения $M$ также указаны в таблице

\begin{tabular}{|c|c|}
\hline$w(l, s, p) ; \theta, \lambda, \pm \nu^{2}, M$ & $r^{0}, r^{-}, r$ \\
\hline $\begin{array}{c}\left(\frac{\left(l+\frac{P}{2}\right)^{2}+\left(s+\frac{Q}{2}\right)^{2}+\left(p+\frac{R}{2}\right)^{2}}{\left(l-\frac{P}{2}\right)^{2}+\left(s-\frac{Q}{2}\right)^{2}+\left(p+\frac{R}{2}\right)^{2}}\right)^{k}, \\
P \neq 0, \quad Q \neq 0 \\
\theta=-\frac{P R}{Q}, \quad \lambda=-\frac{P\left(Q^{2}+R^{2}\right)}{2 Q}, \\
\pm \nu^{2}=\frac{P^{2}-Q^{2}-R^{2}}{4}, \quad M=2 k P\end{array}$ & $\begin{array}{l}r^{0}=\left(l^{2}+s^{2}+\left(p+\frac{Q^{2}+R^{2}}{2 R}\right)^{2}\right. \\
\left.-\frac{P^{2} R^{2}+Q^{4}+Q^{2} R^{2}}{4 R^{2}}\right)^{2} \text { при } R \neq 0 ; \\
r^{0}=p^{2} \text { при } R=0 ; \\
r^{-}=\left(l^{2}+s^{2}+p^{2}\right. \\
\left.\quad-\frac{P^{2}}{Q^{2}} R p-\frac{P^{2}+Q^{2}+R^{2}}{4}-\frac{P^{2} R^{2}}{2 Q^{2}}\right)^{2} \\
+\frac{P^{2} R^{2}+Q^{4}+Q^{2} R^{2}}{Q^{4}}(-P s+Q l)^{2}\end{array}$ \\
\hline $\begin{array}{c}\exp \left(2 k \operatorname{arctg} \frac{P l+Q s}{l^{2}+s^{2}+\left(p+\frac{R}{2}\right)^{2}-\frac{P^{2}+Q^{2}}{4}}\right) \\
P \neq 0, \quad Q \neq 0 \\
\theta=-\frac{P R}{Q}, \quad \lambda=\frac{P\left(Q^{2}-R^{2}\right)}{2 Q}, \\
\pm \nu^{2}=\frac{P^{2}-Q^{2}+R^{2}}{4}, \quad M=2 k P\end{array}$ & $\begin{array}{l}r^{0}=\left(l^{2}+s^{2}+\left(p+\frac{R^{2}-Q^{2}}{2 R}\right)^{2}\right. \\
\left.\quad+\frac{P^{2} R^{2}-Q^{4}+Q^{2} R^{2}}{4 R^{2}}\right)^{2} \text { при } R \neq 0 \\
r^{0}=p^{2} \text { при } R=0 ; \\
r^{-}=\left(l^{2}+s^{2}+p^{2}+R p\right. \\
\left.\quad-\frac{P^{2}+Q^{2}-R^{2}}{4}\right)^{2}+(Q s-P l)^{2}\end{array}$ \\
\hline$\frac{\exp \frac{k(\theta l-2 \nu s)}{l^{2}+s^{2}+(p+\nu)^{2}}}{\lambda=\theta \nu, \quad M=2 k \theta}$ & $\begin{aligned} r^{0} & =\left(l^{2}+s^{2}+(p+\nu)^{2}\right)^{2} \\
r^{-} & =\left(2 \nu\left(l^{2}+s^{2}+p^{2}-\nu^{2}\right)-\theta^{2}(p+\nu)\right)^{2} \\
& +\left(\theta^{2}+4 \nu^{2}\right)(\theta s+2 \nu l)^{2}\end{aligned}$ \\
\hline$\left(\frac{(l+\nu)^{2}+s^{2}+p^{2}}{(l-\nu)^{2}+s^{2}+p^{2}}\right)^{k}$ & $\begin{array}{l}r^{0}=s^{2}+p^{2} ; \\
r^{-}=\left(l^{2}+s^{2}+p^{2}-\nu^{2}\right)^{2} ; \\
r=\operatorname{arctg} \frac{s}{p}+\frac{\theta}{2 \nu} \ln \frac{l^{2}+s^{2}+(p+\nu)^{2}}{\sqrt{s^{2}+p^{2}}}\end{array}$ \\
\hline
\end{tabular}


ТАБлицА 8 (продолжение)

\begin{tabular}{|c|c|}
\hline$w(l, s, p) ; \theta, \lambda, \pm \nu^{2}, M$ & $r^{0}, r^{-}, r$ \\
\hline$\frac{\exp \left(2 k \operatorname{arctg} \frac{2 \nu l}{l^{2}+s^{2}+p^{2}-\nu^{2}}\right)}{\lambda=0, \quad M=4 k \nu}$ & $\begin{array}{l}r^{0}=s^{2}+p^{2} \\
r^{-}=\left(l^{2}+s^{2}+p^{2}+\nu^{2}\right)^{2} \\
r=\operatorname{arctg} \frac{s}{p} \\
-\frac{\theta}{2 \nu} \arcsin \left(\frac{l^{2}+s^{2}+p^{2}-\nu^{2}}{\sqrt{\left(l^{2}+s^{2}+p^{2}+\nu^{2}\right)^{2}-4 \nu^{2}\left(s^{2}+p^{2}\right)}}\right)\end{array}$ \\
\hline $\begin{array}{c}\exp \frac{k l}{l^{2}+s^{2}+p^{2}} \\
\lambda=0, \quad \nu=0, \quad M=k\end{array}$ & $\begin{array}{l}r^{0}=s^{2}+p^{2} ; \\
r^{-}=\left(l^{2}+s^{2}+p^{2}\right)^{2} ; \\
r=\operatorname{arctg} \frac{s}{p}+\frac{\theta l}{l^{2}+s^{2}+p^{2}}\end{array}$ \\
\hline
\end{tabular}

ТАБлицА 9. Семейства из раздела III: $v=v(x, y, z)$ или $v=v(l, s, p)$, где $(l, s, p)$ - выбранная подходящим образом ортогональная система координат; всюду произвольные константы $-\Delta$ и $L$

\begin{tabular}{|c|c|}
\hline$v(x, y, z)(=v(l, s, p))$ & Алгебра Ли $\Xi$ \\
\hline$l^{1+k} V\left(\frac{l^{2}}{s^{2}+p^{2}}, D \operatorname{arctg} \frac{s}{p}-\ln l\right)$ & $\Delta\left(D\left(\Xi_{*}-k \psi \partial_{\psi}\right)+\left(p \partial_{s}-s \partial_{p}\right)\right)+L \partial_{\psi}$ \\
\hline$e^{-k l} V\left(s^{2}+p^{2}, l-\lambda \operatorname{arctg} \frac{s}{p}\right)$ & $\Delta\left(\lambda\left(\partial_{l}-k \psi \partial_{\psi}\right)+\left(p \partial_{s}-s \partial_{p}\right)\right)+L \partial_{\psi}$ \\
\hline $\exp \left(-k \operatorname{arctg} \frac{s}{p}\right) V\left(s^{2}+p^{2}, l\right)$ & $\Delta\left(p \partial_{s}-s \partial_{p}+k \psi \partial_{\psi}\right)+L \partial_{\psi}$ \\
\hline $\begin{array}{c}V(x, y, z)-\text { однородная } \\
\text { функция степени }(1+k)\end{array}$ & $\Delta\left(\Xi_{*}-k \psi \partial_{\psi}\right)+L \partial_{\psi}$ \\
\hline $\begin{array}{c}\exp (k(H x+I y+J z)) V(I x-H y, J x-H z) \\
\text { при } H \neq 0, \\
\text { в противном случае один из аргументов } \\
\text { заменяется на } J y-I z\end{array}$ & $\Delta\left(\Xi_{\uparrow}(H, I, J)-k \psi \partial_{\psi}\right)+L \partial_{\psi}$ \\
\hline
\end{tabular}

доказывать, что независимость $v$ от $\psi$ означает независимость от $\psi$ коэффициентов в выражении для $v$. Этот - четвертый - этап хотя и обосновывает достаточно “очевидный” факт, оказьвается достаточно кропотливым и трудоемким: каждый случай требует своих приемов.

Поскольку в дальнейшем анализе постоянно возникают случаи функции $v(x, y, z)$ вида (I.1)-(I.5), мы заранее исследуем эти “особые" случаи, для того чтобы потом к ним не возврашаться. Это - пятый этап (правда, в нашем изложении, в целях удобства, он предшествует четвертому). 
Последний, шестой этап - уже детальная, “тонкая” классификация. В ее основе лежит решение специального уравнения

$$
-\left(\frac{S^{\prime \prime}(r)}{S^{\prime}(r)} \frac{1}{\chi^{\prime}(r)}+\left(\frac{1}{\chi^{\prime}(r)}\right)^{\prime}\right)=\lambda S(r)+\mu
$$

которое в зависимости от значений $\lambda$ и $\mu$ приводит к шести вариантам зависимости $S(\chi(r))$ (и, соответственно, функции $v(x, y, z)$ ). Именно эти шесть вариантов и просматриваются в таблицах $3-7$, хотя есть и исключения: некоторые варианты оказьваются функциями вида (I.1)-(I.5), а у плоскослоистого варианта $v(x, y, z)=V(P x+Q y+R z)$ появляется одно "степенное" подсемейство с пятимерной группой симметрий, аналогов которого нет у других семейств.

Отметим, что с аналитической точки зрения практически все подсемейства одного семейства эквивалентны: с этой точки зрения нет разницы между обычными тригонометрическими и гиперболическими функциями. Точно так же в семействе осесимметричных $v(x, y, z)$ можно было бы не различать $+\nu^{2}$ и $-\nu^{2}$. Однако оказывается, что, будучи аналитически эквивалентными, геометрически они оказываются принципиально различными. Уже случай простейших функций (I.3)-(I.5) показывает, что в зависимости от знака перед $\nu^{2}$ геометрия лучей оказывается либо сферической, либо Лобачевского, либо евклидовой (см. 7 ). Поэтому мы в дальнейшем предпочитаем сохранять вешественные термины и усматривать различие в перечисленных нами вариантах функций скорости $v(x, y, z)$.

\section{§2. Определяющие уравнения}

2.1. Геометрическое условие инвариантности и продолжение алгебры Ли. Для аккуратного доказательства уточним, как мы обещали, смысл условия инвариантности уравнения относительно группы преобразований, следуя при этом традиционным терминам и обозначениям [12]-[14].

Прежде всего, уравнение отождествляется с геометрической поверхностью в "расширенном" пространстве - пространстве переменных и производных (в нашем случае - это семимерное пространство $\left.\left(x, y, z, \psi, \psi_{x}, \psi_{y}, \psi_{z}\right)\right)$. Такая интерпретация позволяет одним геометрическим объектом охватить все многообразие аналитических форм, описывающих одно и то же, по сушеству, уравнение.

Далее, любое преобразование переменных в исходном пространстве порождает преобразование переменных и в расширенном пространстве, преобразование производных вычисляется по обычньм правилам анализа. Преобразование в расширенном пространстве называют продолжением исходного преобразования. Соответственно в расширенное пространство продолжается и векторное поле, порождающее однопараметрическую подгруппу преобразований, и алгебра Ли многопараметрической группы. Это продолжение, обозначаемое обычно через $\Xi^{(1)}$ (если расширенное пространство содержит только первые производные), имеет вид

$$
\Xi^{(1)}=\Xi+\varphi^{x} \partial_{\psi_{x}}+\varphi^{y} \partial_{\psi_{y}}+\varphi^{z} \partial_{\psi_{z}}
$$


где коэффициенты $\varphi^{\alpha}$ вычисляются по формулам

$$
\begin{aligned}
& \varphi^{x}=\varphi_{x}+\left(\varphi_{\psi}-\xi_{x}\right) \psi_{x}-\eta_{x} \psi_{y}-\zeta_{x} \psi_{z}-\xi_{\psi} \psi_{x}^{2}-\eta_{\psi} \psi_{x} \psi_{y}-\zeta_{\psi} \psi_{x} \psi_{z} \\
& \varphi^{y}=\varphi_{y}+\left(\varphi_{\psi}-\eta_{y}\right) \psi_{y}-\xi_{y} \psi_{x}-\zeta_{y} \psi_{z}-\eta_{\psi} \psi_{y}^{2}-\xi_{\psi} \psi_{x} \psi_{y}-\zeta_{\psi} \psi_{y} \psi_{z} \\
& \varphi^{z}=\varphi_{z}+\left(\varphi_{\psi}-\zeta_{z}\right) \psi_{z}-\xi_{z} \psi_{x}-\eta_{z} \psi_{y}-\zeta_{\psi} \psi_{z}^{2}-\xi_{\psi} \psi_{x} \psi_{z}-\eta_{\psi} \psi_{y} \psi_{z}
\end{aligned}
$$

Язык расширенного пространства удобен еше и тем, что, определив продолжение векторного поля и алгебры Ли, мы можем просто “забыть" про то, что $\psi_{x}, \psi_{y}, \psi_{z}$ - не свободные переменные, а производные (их "происхождение" уже "зашито" в формулы (3)), и сосредоточиться на условии инвариантности геометрической поверхности в расширенном пространстве относительно группы преобразований в этом расширенном пространстве.

Последнее же условие эквивалентно тому, что соответствующая алгебра Ли состоит из векторных полей, касательных к поверхности.

Таким образом, условие инвариантности уравнения относительно группы преобразований сводится к условию, что продолжение соответствующей алгебры Ли состоит из векторных полей, касательных к поверхности в расширенном пространстве, задающей уравнение. Если поверхность задана уравнением

$$
F\left(x, y, z, \psi, \psi_{x}, \psi_{y}, \psi_{z}\right)=0
$$

то аналитически условие инвариантности записьвается как $\left.\Xi^{(1)} F\right|_{F=0}=0$. Именно этим условием мы и воспользуемся.

2.2. Определяющие уравнения. В нашем случае $F=\psi_{x}^{2}+\psi_{y}^{2}+\psi_{z}^{2}-$ $1 / v^{2}(x, y, z)$ и условие инвариантности приобретает вид

$$
2 \varphi^{x} \psi_{x}+2 \varphi^{y} \psi_{y}+2 \varphi^{z} \psi_{z}+2 \xi \frac{v_{x}}{v^{3}}+2 \eta \frac{v_{y}}{v^{3}}+2 \zeta \frac{v_{z}}{v^{3}}=0
$$

что после подстановки формул (3) приводит к уравнению

$$
\begin{aligned}
& \varphi_{x} \psi_{x}+\left(\varphi_{\psi}-\xi_{x}\right) \psi_{x}^{2}-\eta_{x} \psi_{x} \psi_{y}-\zeta_{x} \psi_{x} \psi_{z}-\xi_{\psi} \psi_{x}^{3}-\eta_{\psi} \psi_{x}^{2} \psi_{y}-\zeta_{\psi} \psi_{x}^{2} \psi_{z} \\
& \quad+\varphi_{y} \psi_{y}+\left(\varphi_{\psi}-\eta_{y}\right) \psi_{y}^{2}-\xi_{y} \psi_{x} \psi_{y}-\zeta_{y} \psi_{y} \psi_{z}-\eta_{\psi} \psi_{y}^{3}-\xi_{\psi} \psi_{x} \psi_{y}^{2}-\zeta_{\psi} \psi_{y}^{2} \psi_{z} \\
& +\varphi_{z} \psi_{z}+\left(\varphi_{\psi}-\zeta_{z}\right) \psi_{z}^{2}-\xi_{z} \psi_{x} \psi_{z}-\eta_{z} \psi_{y} \psi_{z}-\zeta_{\psi} \psi_{z}^{3}-\xi_{\psi} \psi_{x} \psi_{z}^{2}-\eta_{\psi} \psi_{y} \psi_{z}^{2} \\
& \quad+\xi \frac{v_{x}}{v^{3}}+\eta \frac{v_{y}}{v^{3}}+\zeta \frac{v_{z}}{v^{3}}=0,
\end{aligned}
$$

которое после группировки слагаемых и использования условия $\psi_{x}^{2}+\psi_{y}^{2}+\psi_{z}^{2}=$ $1 / v^{2}$ упрощается до вида

$$
\begin{aligned}
& -\xi_{x} \psi_{x}^{2}-\eta_{y} \psi_{y}^{2}-\zeta_{z} \psi_{z}^{2}-\left(\eta_{x}+\xi_{y}\right) \psi_{x} \psi_{y}-\left(\zeta_{x}+\xi_{z}\right) \psi_{x} \psi_{z}-\left(\eta_{z}+\zeta_{y}\right) \psi_{y} \psi_{z} \\
& +\psi_{x}\left(\varphi_{x}-\xi_{\psi} \frac{1}{v^{2}}\right)+\psi_{y}\left(\varphi_{y}-\eta_{\psi} \frac{1}{v^{2}}\right)+\psi_{z}\left(\varphi_{z}-\zeta_{\psi} \frac{1}{v^{2}}\right) \\
& +\varphi_{\psi} \frac{1}{v^{2}}+\xi \frac{v_{x}}{v^{3}}+\eta \frac{v_{y}}{v^{3}}+\zeta \frac{v_{z}}{v^{3}}=0 .
\end{aligned}
$$


При фиксированных $(x, y, z)$ наше уравнение означает, что некоторая квадратичная функция от $\psi_{x}, \psi_{y}, \psi_{z}$ на сфере $\psi_{x}^{2}+\psi_{y}^{2}+\psi_{z}^{2}-1 / v^{2}=0$ равна нулю. Это возможно тогда и только тогда, когда она пропорциональна функции, определяющей сферу, что дает нам уравнения

$$
\begin{gathered}
\xi_{x}=\eta_{y}=\zeta_{z}=\varphi_{\psi}+\frac{1}{v}\left(\xi v_{x}+\eta v_{y}+\zeta v_{z}\right), \\
\eta_{x}+\xi_{y}=0, \quad \zeta_{x}+\xi_{z}=0, \quad \eta_{z}+\zeta_{y}=0, \\
\varphi_{x}=\frac{\xi_{\psi}}{v^{2}}, \quad \varphi_{y}=\frac{\eta_{\psi}}{v^{2}}, \quad \varphi_{z}=\frac{\zeta_{\psi}}{v^{2}} .
\end{gathered}
$$

Это и есть требуемые определяющие уравнения для компонент $\xi, \eta, \zeta, \varphi$. Начиная с этого момента мы, по сушеству, будем решать систему (4)-(6), находя те условия на $v(x, y, z)$, при которых эта система имеет решение $\xi, \eta, \zeta, \varphi$ с тем или иньм количеством произвольных постоянных.

\section{§3. Первичная классификация}

3.1. Зависимость $\xi, \eta, \zeta$ от $(x, y, z)$. Исследуем сначала зависимость $\xi, \eta, \zeta$ от переменных $x, y, z$. Эта зависимость определяется пятью уравнениями: первыми двумя равенствами из (4) $\xi_{x}=\eta_{y}=\zeta_{z}$ и уравнениями (5). В совокупности они образуют систему условий, определяющих алгебру Лигруппы конформных преобразований пространства $\mathbb{R}^{3}$. Решение этой системы имеет вид (см., например, [15; $\S 9$, п. 3], подробное обоснование можно также найти в [16])

$$
\begin{aligned}
\xi= & A(\psi)\left(x^{2}-y^{2}-z^{2}\right)+2 B(\psi) x y+2 C(\psi) x z+D(\psi) x \\
& +G(\psi) y-F(\psi) z+H(\psi), \\
\eta= & B(\psi)\left(y^{2}-x^{2}-z^{2}\right)+2 A(\psi) x y+2 C(\psi) y z-G(\psi) x \\
& +D(\psi) y+E(\psi) z+I(\psi), \\
\zeta= & C(\psi)\left(z^{2}-x^{2}-y^{2}\right)+2 A(\psi) x z+2 B(\psi) y z+F(\psi) x \\
& -E(\psi) y+D(\psi) z+J(\psi) .
\end{aligned}
$$

В нашем случае, когда коэффициенты зависят от $\psi$, очевидно, речь идет об алгебре несколько более широкой, по сравнению с конформной, группы.

3.2. Функция $\omega(x, y, z)$. Рассмотрим теперь соотношения (6). Их правые части представляют собой компоненты градиента функции $\varphi$. А для существования функции $\varphi$ с заданным градиентом необходимо и достаточно выполнение условий

$$
\left(\frac{\xi_{\psi}}{v^{2}}\right)_{y}=\left(\frac{\eta_{\psi}}{v^{2}}\right)_{x}, \quad\left(\frac{\xi_{\psi}}{v^{2}}\right)_{z}=\left(\frac{\zeta_{\psi}}{v^{2}}\right)_{x}, \quad\left(\frac{\eta_{\psi}}{v^{2}}\right)_{z}=\left(\frac{\zeta_{\psi}}{v^{2}}\right)_{y} .
$$

Нам удобно будет обозначить через $\omega(x, y, z)$ функцию $\omega(x, y, z)=\ln v^{2}(x, y, z)$. Тогда наши условия запишутся в виде системы линейных неоднородных уравнений с частными производными:

$$
\begin{aligned}
\eta_{\psi} \omega_{x}-\xi_{\psi} \omega_{y} & =\eta_{\psi x}-\xi_{\psi y} \\
-\zeta_{\psi} \omega_{x}+\xi_{\psi} \omega_{z} & =-\zeta_{\psi x}+\xi_{\psi z} \\
\zeta_{\psi} \omega_{y}-\eta_{\psi} \omega_{z} & =\zeta_{\psi y}-\eta_{\psi z}
\end{aligned}
$$


Эта система играет очень важную роль в наших рассмотрениях: именно она определяет "баланс" между условиями на скорость $v(x, y, z)$ (а значит, на $\omega(x, y, z)$ ) и условиями на компоненты $\xi, \eta, \zeta$ алгебры Ли. В этом параграфе мы снова будем считать временно $\psi$ просто параметром, а $\xi_{\psi}, \eta_{\psi}, \zeta_{\psi}$ - просто обозначениями трех функций, зависяших, кроме $(x, y, z)$, еше и от параметра $\psi$. Отметим, что система (8) является алгебраически вырожденной (как система относительно $\omega_{x}$, $\left.\omega_{y}, \omega_{z}\right)$ : сложение уравнений с коэффициентами $\zeta_{\psi}, \eta_{\psi}, \xi_{\psi}$ соответственно даст в левой части нуль. Поэтому необходимьм условием ее разрешимости является обращение в нуль соответствуюшей комбинации правых частей. Позже мы покажем, что это условие и достаточно, предъявив решения системы в явном виде.

3.3. Условие разрешимости. Итак, условие разрешимости системы (8) имеет вид

$$
\left(\eta_{\psi x}-\xi_{\psi y}\right) \zeta_{\psi}+\left(-\zeta_{\psi x}+\xi_{\psi z}\right) \eta_{\psi}+\left(\zeta_{\psi y}-\eta_{\psi z}\right) \xi_{\psi}=0
$$

Подстановка в него функций $\xi, \eta, \zeta$ из (7) (при этом для краткости записи мы аргумент $\psi$ у коэффициентов будем опускать, а производные по этому аргументу будем обозначать штрихом) дает уравнение

$$
\begin{aligned}
& 2\left(2 A^{\prime} y-2 B^{\prime} x-G^{\prime}\right)\left(C^{\prime}\left(z^{2}-x^{2}-y^{2}\right)+2 A^{\prime} x z+2 B^{\prime} y z+F^{\prime} x-E^{\prime} y+D^{\prime} z+J^{\prime}\right) \\
& \quad+2\left(2 C^{\prime} x-2 A^{\prime} z-F^{\prime}\right)\left(B^{\prime}\left(y^{2}-x^{2}-z^{2}\right)+2 A^{\prime} x y+2 C^{\prime} y z-G^{\prime} x\right. \\
& \left.\quad+D^{\prime} y+E^{\prime} z+I^{\prime}\right)+2\left(2 B^{\prime} z-2 C^{\prime} y-E^{\prime}\right)\left(A^{\prime}\left(x^{2}-y^{2}-z^{2}\right)\right. \\
& \left.\quad+2 B^{\prime} x y+2 C^{\prime} x z+D^{\prime} x+G^{\prime} y-F^{\prime} z+H^{\prime}\right)=0
\end{aligned}
$$

которое после приведения подобных (кубические члены сокращаются) и приравнивания по отдельности квадратичных, линейных и свободных членов приводится к системе

$$
\begin{aligned}
& \left(2 A^{\prime} y-2 B^{\prime} x\right)\left(F^{\prime} x-E^{\prime} y\right)+\left(2 C^{\prime} x-2 A^{\prime} z\right)\left(-G^{\prime} x+E^{\prime} z\right) \\
& \quad+\left(2 B^{\prime} z-2 C^{\prime} y\right)\left(G^{\prime} y-F^{\prime} z\right)-2\left(A^{\prime} x+B^{\prime} y+C^{\prime} z\right)\left(G^{\prime} z+F^{\prime} y+E^{\prime} x\right) \\
& \quad+\left(A^{\prime} E^{\prime}+B^{\prime} F^{\prime}+C^{\prime} G^{\prime}\right)\left(x^{2}+y^{2}+z^{2}\right)=0, \\
& \left(2 A^{\prime} y-2 B^{\prime} x\right) J^{\prime}+\left(2 C^{\prime} x-2 A^{\prime} z\right) I^{\prime}+\left(2 B^{\prime} z-2 C^{\prime} y\right) H^{\prime} \\
& \quad-D^{\prime}\left(E^{\prime} x+F^{\prime} y+G^{\prime} z\right)=0, \\
& E^{\prime} H^{\prime}+F^{\prime} I^{\prime}+G^{\prime} J^{\prime}=0 .
\end{aligned}
$$

Из этой системы мы получаем условия на коэффициенты:

$$
\begin{gathered}
A^{\prime} E^{\prime}+B^{\prime} F^{\prime}+C^{\prime} G^{\prime}=0 \\
-2 B^{\prime} J^{\prime}+2 C^{\prime} I^{\prime}=D^{\prime} G^{\prime}, \quad 2 A^{\prime} J^{\prime}-2 C^{\prime} H^{\prime}=D^{\prime} F^{\prime}, \\
-2 A^{\prime} I^{\prime}+2 B^{\prime} H^{\prime}=D^{\prime} E^{\prime} \\
E^{\prime} H^{\prime}+F^{\prime} I^{\prime}+G^{\prime} J^{\prime}=0
\end{gathered}
$$

являющиеся основой первичной классификации. 
3.4. Случай $A^{\prime 2}+B^{\prime 2}+C^{\prime 2} \neq 0$. В этом случае за счет сдвига системы координат $\widetilde{x}=x-\alpha(\psi), \widetilde{y}=y-\beta(\psi), \widetilde{z}=z-\gamma(\psi)$ удается сделать коэффициенты $D^{\prime}, E^{\prime}, F^{\prime}, G^{\prime}$ нулевыми (при этом играет роль то, что в силу условия (9) вектор $\left(E^{\prime}, F^{\prime}, G^{\prime}\right)$ ортогонален вектору $\left.\left(A^{\prime}, B^{\prime}, C^{\prime}\right)\right)$. Тогда уравнения $(9)$ и (11) оказываются выполненными автоматически, а уравнения (10) означают пропорциональность вектора $\left(H^{\prime}, I^{\prime}, J^{\prime}\right)$ вектору $\left(A^{\prime}, B^{\prime}, C^{\prime}\right)$. Если коэффициент пропорциональности обозначить через $\mp \nu^{2}$, то в новой системе координат

$$
\begin{aligned}
\xi_{\psi} & =A^{\prime}\left(\widetilde{x}^{2}-\widetilde{y}^{2}-\widetilde{z}^{2} \mp \nu^{2}\right)+2 B^{\prime} \widetilde{x} \widetilde{y}+2 C^{\prime} \widetilde{x} \widetilde{z} \\
\eta_{\psi} & =B^{\prime}\left(\widetilde{y}^{2}-\widetilde{x}^{2}-\widetilde{z}^{2} \mp \nu^{2}\right)+2 A^{\prime} \widetilde{x} \widetilde{y}+2 C^{\prime} \widetilde{y} \widetilde{z} \\
\zeta_{\psi} & =C^{\prime}\left(\widetilde{z}^{2}-\widetilde{y}^{2}-\widetilde{x}^{2} \mp \nu^{2}\right)+2 B^{\prime} \widetilde{y} \widetilde{z}+2 A^{\prime} \widetilde{x} \widetilde{z}
\end{aligned}
$$

Обратимся теперь к системе (8). Соответствуюшая однородная система

$$
\eta_{\psi} \omega_{x}-\xi_{\psi} \omega_{y}=0, \quad-\zeta_{\psi} \omega_{x}+\xi_{\psi} \omega_{z}=0, \quad \zeta_{\psi} \omega_{y}-\eta_{\psi} \omega_{z}=0
$$

решается методом характеристик. Первое уравнение имеет характеристику, определяемую системой

$$
\frac{d x}{\eta_{\psi}}=-\frac{d y}{\xi_{\psi}}=\frac{d z}{0}
$$

имеющей для $\xi, \eta, \zeta$ вида (12) два первых интеграла $z=\mathrm{const}, l / \sigma=\mathrm{const}$ (далее для удобства обозначено $l=A^{\prime} \widetilde{x}+B^{\prime} \widetilde{y}+C^{\prime} \widetilde{z}, \sigma=\widetilde{x}^{2}+\widetilde{y}^{2}+\widetilde{z}^{2} \pm \nu^{2}$ ). Поэтому общее решение первого уравнения имеет вид $\omega(x, y, z)=\Omega(l / \sigma, z)$, где $\Omega(\cdot)$ - произвольная непрерывно дифференцируемая функция. Подстановка этой функции в остальные два уравнения дает $\Omega_{z}=0$, так что обшее решение системы имеет вид $\omega(x, y, z)=\Omega(l / \sigma)$. Поиск частного решения неоднородной системы в виде $\omega(x, y, z)=\omega(l, \sigma)$ приводит к одному-единственному уравнению $l \omega_{l}+\sigma \omega_{\sigma}=2$, имеюшему очевидное решение, например $\omega(l, \sigma)=2 \ln \sigma$.

Таким образом, общее решение системы (8) в предположении $A^{\prime 2}+B^{\prime 2}+C^{\prime 2} \neq 0$ оказалось равным $\omega(x, y, z)=2 \ln \sigma+\Omega(l / \sigma)$, что соответствует функции скорости

$$
v(x, y, z)=\sigma \exp \left(\frac{1}{2} \Omega\left(\frac{l}{\sigma}\right)\right)=\sigma V\left(\frac{l}{\sigma}\right)
$$

где $V$ - произвольная функция от $r=l / \sigma$. Мы получили семейство (II.4). Поскольку врашения пространства вокруг оси $\left(A^{\prime}, B^{\prime}, C^{\prime}\right)$ не меняют ни значения $l$, ни значения $\sigma$, поверхности уровня функции $v(x, y, z)$ наверняка являются фигурами врашения вокруг этой оси. Таким образом, полученная нами функция скорости соответствует осесимметрично-слоистой среде.

Зафииксировав полученное решение, мы на время оставим его для того, чтобы довести до конца первичную классификацию.

3.5. Случай $A^{\prime}=B^{\prime}=C^{\prime}=0, E^{2}+F^{2}+G^{\prime 2} \neq 0$. В этом случае уравнение (9) обрашается в тождество, из уравнений (10) получаем $D^{\prime}=0$, а из условия (11) ортогональности вектора $\left(H^{\prime}, I^{\prime}, J^{\prime}\right)$ вектору $\left(E^{\prime}, F^{\prime}, G^{\prime}\right)$ следует 
возможность за счет сдвига системы координат сделать $H^{\prime}, I^{\prime}, J^{\prime}$ нулями. Таким образом, мы получаем

$$
\xi_{\psi}=G^{\prime} \widetilde{y}-F^{\prime} \widetilde{z}, \quad \eta_{\psi}=-G^{\prime} \widetilde{x}+E^{\prime} \widetilde{z}, \quad \zeta_{\psi}=F^{\prime} \widetilde{x}-E^{\prime} \widetilde{y}
$$

Теперь интегрирование системы (8) уже не представляет труда: соответствуюшая однородная система

$$
\eta_{\psi} \omega_{x}-\xi_{\psi} \omega_{y}=-\zeta_{\psi} \omega_{x}+\xi_{\psi} \omega_{z}=\zeta_{\psi} \omega_{y}-\eta_{\psi} \omega_{z}=0
$$

имеет для $\xi, \eta, \zeta$ вида (14) (в предположении $G^{\prime} \neq 0$, не ограничиваюшем обшности) решение $\omega(x, y, z)=\Omega\left(\left(G^{\prime} \widetilde{y}-F^{\prime} \widetilde{z}\right) /\left(-G^{\prime} \widetilde{x}+E^{\prime} \widetilde{z}\right)\right)$, где $\Omega$ - произвольная непрерывно дифференцируемая функция, а решение неоднородной системы удается, как и в предыдушем случае, отыскать в виде $\omega(x, y, z)=2 \ln \left|-G^{\prime} \widetilde{x}+E^{\prime} \widetilde{z}\right|$. Таким образом, обшее решение системы (8) в случае $\xi, \eta, \zeta$ вида (14) равно

$$
\omega(x, y, z)=2 \ln \left|-G^{\prime} \widetilde{x}+E^{\prime} \widetilde{z}\right|+\Omega\left(\frac{G^{\prime} \widetilde{y}-F^{\prime} \widetilde{z}}{-G^{\prime} \widetilde{x}+E^{\prime} \widetilde{z}}\right),
$$

а функция скорости -

$$
v(x, y, z)=\left(-G^{\prime} \widetilde{x}+E^{\prime} \widetilde{z}\right) V\left(\frac{G^{\prime} \widetilde{y}-F^{\prime} \widetilde{z}}{-G^{\prime} \widetilde{x}+E^{\prime} \widetilde{z}}\right) .
$$

Мы получили семейство (ІІ.3). Найденная нами функция скорости соответствует среде с цилиндрическим слоением: линии уровня функции $v(x, y, z)$ являются цилиндрами с направляющими, параллельными вектору $\left(E^{\prime}, F^{\prime}, G^{\prime}\right)$, причем эти цилиндры подобны друг другу (гомотетия с коэффициентом $k$ переводит поверхность $v=1$ в поверхность $v=k)$.

3.6. Случай $A^{\prime}=B^{\prime}=C^{\prime}=E^{\prime}=F^{\prime}=G^{\prime}=0, D^{\prime} \neq 0$. Здесь все уравнения (9)-(11) оказьваются тождествами; за счет сдвига системы координат получаем $\xi_{\psi}=D^{\prime} \widetilde{x}, \eta_{\psi}=D^{\prime} \widetilde{y}, \zeta_{\psi}=D^{\prime} \widetilde{z}$, система (8) оказывается однородной, и ее общее решение имеет вид $\omega(x, y, z)=\Omega\left(\sigma_{0}\right)$, где $\sigma_{0}=\widetilde{x}^{2}+\widetilde{y}^{2}+\widetilde{z}^{2}$, а $\Omega$ произвольная непрерьвно дифференцируемая функция. Соответственно функция скорости имеет вид

$$
v(x, y, z)=V\left(\sigma_{0}\right)=V\left(x^{2}+y^{2}+z^{2}\right),
$$

что дает нам семейство (II.2), описываюшее сферически слоистые среды.

3.7. Случай $A^{\prime}=B^{\prime}=C^{\prime}=E^{\prime}=F^{\prime}=G^{\prime}=D^{\prime}=0, H^{\prime 2}+I^{\prime 2}+$ $J^{\prime 2} \neq 0$. Здесь $\xi_{\psi}=H^{\prime}, \eta_{\psi}=I^{\prime}, \zeta_{\psi}=J^{\prime}$, обшее решение системы (8) имеет вид $\omega(x, y, z)=\Omega(s)$, где $s=H^{\prime} x+I^{\prime} y+J^{\prime} z$, а $\Omega$ - произвольная непрерьвно дифференцируемая функция. Функция скорости соответственно имеет вид

$$
v(x, y, z)=V(s)=V\left(H^{\prime} x+I^{\prime} y+J^{\prime} z\right),
$$

что соответствует семейству (II.1), описываюшему плоско-слоистые среды. 
3.8. Случай $\xi_{\psi}=\eta_{\psi}=\zeta_{\psi} \equiv 0$. В этом случае система уравнений (8) превращается в тождество - значит, ей удовлетворяет любая функция $\omega(x, y, z)$ и любая функция скорости. Более детальный анализ этого случая, как и остальных, будет произведен позже - там появятся дополнительные ограничения на $\omega$ и $v$.

\section{§4. Особые случаи функции скорости (I.1)-(I.5)}

Поскольку в дальнейших рассуждениях нам постоянно будут встречаться в качестве частных случаев функции скорости (I.1)-(I.5), нам будет удобно заранее описать алгебры Ли соответствуюших групп симметрий, чтобы потом просто исключать соответствуюшие частные случаи из рассмотрения.

4.1. Случай $v(x, y, z) \equiv v=$ const. В этом случае $\omega(x, y, z)=$ const и уравнения (8) дают нам условия $\xi_{\psi y}=\eta_{\psi x}, \zeta_{\psi x}=\xi_{\psi z}, \eta_{\psi z}=\zeta_{\psi y}$, из которых ввиду условий (5) получаем

$$
\xi_{\psi y}=\eta_{\psi x}=\zeta_{\psi x}=\xi_{\psi z}=\eta_{\psi z}=\zeta_{\psi y}=0
$$

откуда $A^{\prime}=B^{\prime}=C^{\prime}=E^{\prime}=F^{\prime}=G^{\prime}=0$, а значит, $A, B, C, D, E, F, G$ являются константами: $A(\psi)=\bar{A}, B(\psi)=\bar{B}, C(\psi)=\bar{C}, E(\psi)=\bar{E}, F(\psi)=\bar{F}, G(\psi)=\bar{G}$.

Система (6) превращается в систему

$$
\varphi_{x}=\frac{\xi_{\psi}}{v^{2}}=\frac{D^{\prime} x+H^{\prime}}{v^{2}}, \quad \varphi_{y}=\frac{\eta_{\psi}}{v^{2}}=\frac{D^{\prime} y+I^{\prime}}{v^{2}}, \quad \varphi_{z}=\frac{\zeta_{\psi}}{v^{2}}=\frac{D^{\prime} z+J^{\prime}}{v^{2}}
$$

и ее решение имеет вид

$$
\varphi(x, y, z, \psi)=\frac{D^{\prime}(\psi)\left(x^{2}+y^{2}+z^{2}\right) / 2+H^{\prime}(\psi) x+I^{\prime}(\psi) y+J^{\prime}(\psi) z}{v^{2}}+\widehat{\varphi}(\psi) .
$$

Подстановка этого решения в последнее из неиспользованных нами пока уравнений $(4)$, которое для $v(x, y, z)=v$ принимает вид $\varphi_{\psi}=\xi_{x}=\eta_{y}=\zeta_{z}$, приводит к уравнению

$$
\begin{aligned}
& \frac{D^{\prime \prime}(\psi)\left(x^{2}+y^{2}+z^{2}\right) / 2+H^{\prime \prime}(\psi) x+I^{\prime \prime}(\psi) y+J^{\prime \prime}(\psi) z}{v^{2}}+\widehat{\varphi}^{\prime}(\psi) \\
& \quad=2(\bar{A} x+\bar{B} y+\bar{C} z)+D(\psi) .
\end{aligned}
$$

Приравнивание коэффициентов дает условия $D^{\prime \prime}=0, H^{\prime \prime}=2 v^{2} \bar{A}, I^{\prime \prime}=2 v^{2} \bar{B}$, $J^{\prime \prime}=2 v^{2} \bar{C}, \widehat{\varphi}^{\prime}=D$, из которых определяются

$$
\begin{gathered}
D=2 D_{1} \psi+D_{2}, \quad \widehat{\varphi}=D_{1} \psi^{2}+D_{2} \psi+L \\
H=A v^{2} \psi^{2}+H_{1} \psi+H_{2}, \quad I=B v^{2} \psi^{2}+I_{1} \psi+I_{2}, \quad J=C v^{2} \psi^{2}+J_{1} \psi+J_{2} .
\end{gathered}
$$


Таким образом, алгебра Ли группы симметрий уравнения эйконала с постоянной скоростью имеет компоненты

$$
\begin{aligned}
\xi(x, y, z, \psi)= & \bar{A}\left(\psi^{2} v^{2}+x^{2}-y^{2}-z^{2}\right)+2 \bar{B} x y+2 \bar{C} x z+\left(2 D_{1} \psi+D_{2}\right) x \\
& +\bar{G} y-\bar{F} z+H_{1} \psi+H_{2} \\
\eta(x, y, z, \psi)= & \bar{B}\left(\psi^{2} v^{2}+y^{2}-x^{2}-z^{2}\right)+2 \bar{A} x y+2 \bar{C} y z+\left(2 D_{1} \psi+D_{2}\right) y \\
& -\bar{G} x+\bar{E} z+I_{1} \psi+I_{2} \\
\zeta(x, y, z, \psi)= & \bar{C}\left(\psi^{2} v^{2}+z^{2}-x^{2}-y^{2}\right)+2 \bar{B} y z+2 \bar{A} x z+\left(2 D_{1} \psi+D_{2}\right) z \\
& +\bar{F} x-\bar{E} y+J_{1} \psi+J_{2} \\
\varphi(x, y, z, \psi)= & \frac{D_{1}\left(\psi^{2} v^{2}+x^{2}+y^{2}+z^{2}\right)+H_{1} x+I_{1} y+J_{1} z}{v^{2}} \\
& +\psi\left(2 \bar{A} x+2 \bar{B} y+2 \bar{C} z+D_{2}\right)+L
\end{aligned}
$$

что в точности дает алгебру, указанную в первой строке таблицы 1.

4.2. Случай $v(x, y, z)=P x+Q y+R z$. Этот случай рассматривается по той же схеме, что и предыдущий, поэтому мы изложим его вкратце, опустив детальные вькладки. В этом случае система (8) приобретает вид

$$
\begin{aligned}
2 P \eta_{\psi}-2 Q \xi_{\psi} & =\left(\eta_{\psi x}-\xi_{\psi y}\right)(P x+Q y+R z), \\
-2 P \zeta_{\psi}+2 R \xi_{\psi} & =\left(-\zeta_{\psi x}+\xi_{\psi z}\right)(P x+Q y+R z), \\
2 Q \zeta_{\psi}-2 R \eta_{\psi} & =\left(\zeta_{\psi y}-\eta_{\psi z}\right)(P x+Q y+R z),
\end{aligned}
$$

и подстановка в эти уравнения функций (7) дает систему соотношений относительно квадратичных функций, из которых приравниванием коэффициентов при одинаковых степенях получаем набор уравнений относительно коэффициентов: $B^{\prime} P=A^{\prime} Q, C^{\prime} P=A^{\prime} R, B^{\prime} R=C^{\prime} Q, D^{\prime}=0, E^{\prime} P+F^{\prime} Q+G^{\prime} R=0, I^{\prime} P=H^{\prime} Q$, $J^{\prime} P=H^{\prime} R, J^{\prime} Q=I^{\prime} R$. Эти соотношения позволяют представить коэффициенты $A, B, C, D, E, F, G$ и $H, I, J$ в виде

$$
\begin{gathered}
A(\psi)=\lambda(\psi) P+\bar{A}, \quad B(\psi)=\lambda(\psi) Q+\bar{B}, \quad C(\psi)=\lambda(\psi) R+\bar{C}, \quad D(\psi) \equiv \bar{D}, \\
E=\widehat{E}(\psi)+\theta P, \quad F=\widehat{F}(\psi)+\theta Q, \quad G=\widehat{G}(\psi)+\theta R \\
H(\psi)=\varkappa(\psi) P+\bar{H}, \quad I(\psi)=\varkappa(\psi) Q+\bar{I}, \quad J(\psi)=\varkappa(\psi) R+\bar{J}
\end{gathered}
$$

где $\bar{A} P+\bar{B} Q+\bar{C} R=0, \widehat{E}(\psi) R+\widehat{F}(\psi) Q+\widehat{G}(\psi) P=0$ и $\bar{H} P+\bar{I} Q+\bar{J} R=0$.

Подстановка $\xi, \eta, \zeta$ с выраженньми вышеприведенньм образом $A, B, C, D, E, F$, $G, H, I, J$ в уравнения (6) позволяет проинтегрировать эти уравнения и получить

$$
\varphi(x, y, z, \psi)=\frac{\lambda^{\prime}\left(x^{2}+y^{2}+z^{2}\right)-\left(\widehat{G}^{\prime} y-\widehat{F}^{\prime} z\right) / P-\varkappa^{\prime}}{P x+Q y+R z}+\widehat{\varphi} .
$$

Подстановка полученной $\varphi$ в соотношение

$$
v \varphi_{\psi}+\left(\xi v_{x}+\eta v_{y}+\zeta v_{z}\right)=v \xi_{x}
$$


приводит опять к равенству двух квадратичных функций, из которого после сокрашений и приведения подобных получаются соотношения

$$
\begin{gathered}
\lambda^{\prime \prime}(\psi)=\left(P^{2}+Q^{2}+R^{2}\right) \lambda(\psi), \quad \varkappa^{\prime \prime}(\psi)=\left(P^{2}+Q^{2}+R^{2}\right) \varkappa(\psi), \\
\widehat{E}^{\prime \prime}(\psi)=\left(P^{2}+Q^{2}+R^{2}\right) \widehat{E}(\psi), \quad \widehat{F}^{\prime \prime}(\psi)=\left(P^{2}+Q^{2}+R^{2}\right) \widehat{F}(\psi), \\
-P \widehat{\varphi}^{\prime}(\psi)=R \widehat{F}(\psi)-Q \widehat{E}(\psi) .
\end{gathered}
$$

После обозначения $w^{2}=P^{2}+Q^{2}+R^{2}$, решения приведенных уравнений и подстановки их в $(7)$ и формулу для $\varphi$ эти соотношения дают как раз формулы второй строки таблицы 1:

$$
\begin{aligned}
\xi= & \left(\lambda_{1} \operatorname{ch} w \psi+\lambda_{2} \operatorname{sh} w \psi\right)\left(P\left(x^{2}-y^{2}-z^{2}\right)+2 Q x y+2 R x z\right) \\
& +\bar{A}\left(x^{2}-y^{2}-z^{2}\right)+2 \bar{B} x y+2 \bar{C} x z+\bar{D} x+\left(G_{1} \operatorname{ch} w \psi+G_{2} \operatorname{sh} w \psi\right) y \\
& -\left(F_{1} \operatorname{ch} w \psi+F_{2} \operatorname{sh} w \psi\right) z+\theta(R y-Q z)+\left(\varkappa_{1} \operatorname{ch} w \psi+\varkappa_{2} \operatorname{sh} w \psi\right) P+\bar{H}, \\
\eta= & \left(\lambda_{1} \operatorname{ch} w \psi+\lambda_{2} \operatorname{sh} w \psi\right)\left(Q\left(y^{2}-x^{2}-z^{2}\right)+2 P x y+2 R y z\right) \\
& +\bar{B}\left(y^{2}-x^{2}-z^{2}\right)+2 \bar{A} x y+2 \bar{C} y z+\bar{D} y-\left(G_{1} \operatorname{ch} w \psi+G_{2} \operatorname{sh} w \psi\right) x \\
& +\left(E_{1} \operatorname{ch} w \psi+E_{2} \operatorname{sh} w \psi z+\theta(-R x+P z)+\left(\varkappa_{1} \operatorname{ch} w \psi+\varkappa_{2} \operatorname{sh} w \psi\right) Q+\bar{I},\right. \\
\zeta= & \left(\lambda_{1} \operatorname{ch} w+\lambda_{2} \operatorname{sh} w \psi\right)\left(R\left(z^{2}-x^{2}-y^{2}\right)+2 P x z+2 Q y z\right) \\
& +\bar{C}\left(z^{2}-x^{2}-y^{2}\right)+2 \bar{A} x z+2 \bar{B} y z+\bar{D} z+\left(F_{1} \operatorname{ch} w \psi+F_{2} \operatorname{sh} w \psi\right) x \\
& -\left(E_{1} \operatorname{ch} w \psi+E_{2} \operatorname{sh} w \psi\right) y+\theta(Q x-P y)+\left(\varkappa_{1} \operatorname{ch} w \psi+\varkappa_{2} \operatorname{sh} w \psi\right) R+\bar{J}, \\
\varphi= & \left(\lambda_{1} \operatorname{sh} w \psi+\lambda_{2} \operatorname{ch} w \psi\right) \frac{x^{2}+y^{2}+z^{2}}{P x+Q y+R z}-\frac{w\left(\varkappa_{1} \operatorname{sh} w \psi+\varkappa_{2} \operatorname{ch} w \psi\right)}{P x+Q y+R z} \\
& +\frac{E_{1}(R y-Q z)+F_{1}(P z-R x)+G_{1}(Q x-P y)}{w(P x+Q y+R z)} \operatorname{sh} w \psi \\
& +\frac{E_{2}(R y-Q z)+F_{2}(P z-R x)+G_{2}(Q x-P y)}{w(P x+Q y+R z)} \operatorname{ch} w \psi+\bar{L} .
\end{aligned}
$$

4.3. Случай $v(x, y, z)=w \sigma=w\left(x^{2}+y^{2}+z^{2} \pm \nu^{2}\right)$. Этот случай объединяет сразу три формулы (І.3)-(І.5). Символ $\pm \nu^{2}$ обозначает один из трех вариантов: $+\nu^{2},-\nu^{2}$ и 0 . Опять же мы следуем уже отлаженной схеме: уравнения (8) - уравнения (6) - последнее уравнение в (4), и в целях экономии места опускаем элементарные, но достаточно громоздкие выкладки.

Уравнения (8) приобретают вид

$$
\begin{gathered}
\eta_{\psi} \frac{4 x}{\sigma}-\xi_{\psi} \frac{4 y}{\sigma}=\eta_{\psi x}-\xi_{\psi y}, \quad-\zeta_{\psi} \frac{4 x}{\sigma}+\xi_{\psi} \frac{4 z}{\sigma}=-\zeta_{\psi x}+\xi_{\psi z} \\
\zeta_{\psi} \frac{4 y}{\sigma}-\eta_{\psi} \frac{4 z}{\sigma}=\zeta_{\psi y}-\eta_{\psi z} .
\end{gathered}
$$

Подстановка в них функций $\xi, \eta, \zeta$ из (7) после упрошений приводит к соотношениям

$$
\begin{aligned}
4 z\left(E^{\prime} x+F^{\prime} y+G^{\prime} z\right)+4\left(I^{\prime} x-H^{\prime} y\right) & =2 G^{\prime}\left(x^{2}+y^{2}+z^{2} \pm \nu^{2}\right), \\
4 y\left(E^{\prime} x+F^{\prime} y+G^{\prime} z\right)+4\left(H^{\prime} z-J^{\prime} x\right) & =2 F^{\prime}\left(x^{2}+y^{2}+z^{2} \pm \nu^{2}\right), \\
4 x\left(E^{\prime} x+F^{\prime} y+G^{\prime} z\right)+4\left(J^{\prime} y-I^{\prime} z\right) & =2 E^{\prime}\left(x^{2}+y^{2}+z^{2} \pm \nu^{2}\right),
\end{aligned}
$$


которые могут выполняться для всех $(x, y, z)$, только если $E^{\prime}=F^{\prime}=G^{\prime}=H^{\prime}=$ $I^{\prime}=J^{\prime}=0$.

Уравнения (6) тогда приобретают вид

$$
\begin{aligned}
& \varphi_{x}=-\frac{1}{w^{2}}\left(\frac{2 A^{\prime} x+2 B^{\prime} y+2 C^{\prime} z+D^{\prime}}{2 \sigma}\right)_{x}, \\
& \varphi_{y}=-\frac{1}{w^{2}}\left(\frac{2 A^{\prime} x+2 B^{\prime} y+2 C^{\prime} z+D^{\prime}}{2 \sigma}\right)_{y}, \\
& \varphi_{z}=-\frac{1}{w^{2}}\left(\frac{2 A^{\prime} x+2 B^{\prime} y+2 C^{\prime} z+D^{\prime}}{2 \sigma}\right)_{z},
\end{aligned}
$$

откуда

$$
\varphi(x, y, z, \psi)=-\frac{2 A^{\prime} x+2 B^{\prime} y+2 C^{\prime} z+D^{\prime}}{2 w^{2} \sigma}+\widehat{\varphi}(\psi) .
$$

Остается последнее уравнение из (4), которое для нашей функции скорости выглядит как

$$
\sigma \xi_{x}=\sigma \varphi_{\psi}+2 x \xi+2 y \eta+2 z \zeta .
$$

Подставляя найденные выше $\xi, \eta, \zeta, \varphi$, получаем уравнение относительно квадратичных функций и, приравнивая соответствующие коэффициенты, получаем

$$
\begin{gathered}
D^{\prime \prime}=\mp 4 w^{2} \nu^{2} D, \quad \widehat{\varphi}^{\prime}=-D(\psi), \\
A^{\prime \prime}=\mp 4 w^{2} \nu^{2} A+2 w^{2} \bar{H}, \quad B^{\prime \prime}=\mp 4 w^{2} \nu^{2} B+2 w^{2} \bar{I}, \quad C^{\prime \prime}=\mp 4 w^{2} \nu^{2} C+2 w^{2} \bar{J} .
\end{gathered}
$$

Решение этих уравнений в каждом из трех вариантов $\pm \nu^{2}=+\nu^{2}, \pm \nu^{2}=-\nu^{2}$ и $\pm \nu^{2}=0$ и подстановка их в формулы (17) и в формулу для $\varphi$ дает алгебры Ли, указанные в 3-5 строках таблицы 1.

\section{§5. Независимость функции скорости от $\psi$. Определение компонент $(\xi, \eta, \zeta, \varphi)$}

Вернемся к исследованию семейств (II.1)-(II.4). В полученных в 33 формулах (13), (15)-(17) функция $v$ выражена как функция не только от $x, y, z$, но и от $\psi$ (как в коэффициентах, фигурируюших в этих формулах и в параметрах $\alpha$, $\beta, \gamma$ сдвига системы координат для приведения к подходящему виду, так и в самом характере зависимости $V(\cdot))$. Однако на самом деле она от $\psi$ не зависит, и это налагает дополнительные ограничения на параметры. Естественное предположение состоит в том, что независимость $v$ от $\psi$ влечет независимость от $\psi$ всех параметров и функции $V(\cdot)$, однако доказательство этого факта в каждом случае требует специальных приемов алгебраического характера. Нам будет удобнее идти от более простых функций скорости к более сложным (т.е. в порядке, указанном в формулировке теоремы 1). Кроме того, удобнее исследовать независимость от $\psi$ функции $\omega$, а не функции $v$.

5.1. С лучай функции (17). В предположении, что $\omega$ имеет вид $\omega(x, y, z, \psi)=$ $\Omega(s, \psi)$, где $s=H^{\prime}(\psi) x+I^{\prime}(\psi) y+J^{\prime}(\psi) z$, условие независимости от $\psi$ имеет вид

$$
\left(H^{\prime \prime}(\psi) x+I^{\prime \prime}(\psi) y+J^{\prime \prime}(\psi) z\right) \Omega_{s}(s, \psi)+\Omega_{\psi}(s, \psi)=0 .
$$


Зафиксируем значения $\psi$ и $s$. Тогда $x, y, z$ лежат на некоторой гиперплоскости. На этой гиперплоскости левая часть нашего уравнения является линейной функцией от $x, y, z$, и эта функция тождественно равна нулю. Значит, эта функция пропорциональна функции, определяющей гиперплоскость, т.е. $H^{\prime \prime}=k^{\prime}(\psi) H^{\prime}$, $I^{\prime \prime}=k^{\prime}(\psi) I^{\prime}, J^{\prime \prime}=k^{\prime}(\psi) J^{\prime}$. Вообще говоря, коэффициент пропорциональности мог бы зависеть и от $\psi$, и от $s$, но, поскольку $H, I, J$ и их производные от $s$ не зависят, остается только зависимость от $\psi$. Представление коэффициента пропорциональности в виде производной некоторой функции $k(\psi)$ выбрано для удобства. Из наших условий следует, что $H^{\prime}=P e^{k(\psi)}, I^{\prime}=Q e^{k(\psi)}, J^{\prime}=R e^{k(\psi)}$, где $P, Q, R$ - некоторые константы (причем нам будет удобно считать для определенности, что $\left.P^{2}+Q^{2}+R^{2}=1\right)$, а тогда

$$
\begin{gathered}
H^{\prime} x+I^{\prime} y+J^{\prime} z=e^{k(\psi)}(P x+Q y+R z) \\
\Omega\left(H^{\prime} x+I^{\prime} y+J^{\prime} z, \psi\right)=\Omega\left(e^{k(\psi)}(P x+Q y+R z), \psi\right)=\widehat{\Omega}(P x+Q y+R z, \psi)
\end{gathered}
$$

(экспоненциальный множитель, зависящий только от $\psi$, просто включен в зависимость от второго аргумента). Для новой же функции $\widehat{\Omega}$ независимость от $\psi$ есть просто независимость от второго аргумента: $\widehat{\Omega}_{\psi}=0$. Значит, в итоге $\omega(x, y, z, \psi)=$ $\Omega(P x+Q y+R z)$, a $v(x, y, z)=V(P x+Q y+R z)$.

Равенство (18) нам будет удобнее представить в виде $H^{\prime}=\theta^{\prime}(\psi) P, I^{\prime}=\theta^{\prime}(\psi) Q$, $J^{\prime}=\theta^{\prime}(\psi) R$, где $\theta^{\prime}(\psi)=e^{k(\psi)}$, а тогда $H=\theta(\psi) P+\bar{H}, \quad I=\theta(\psi) Q+\bar{I}$, $J=\theta(\psi) R+\bar{J}$, причем можно для определенности выбора $\theta(\psi)$ считать, что $P \bar{H}+Q \bar{I}+R \bar{J}=0$. Вместе с предположениями этого случая $A^{\prime}=B^{\prime}=C^{\prime}=$ $D^{\prime}=E^{\prime}=F^{\prime}=G^{\prime}=0$ это дает описание первых трех компонент алгебры Ли в виде

$$
\begin{aligned}
\xi(x, y, z, \psi)= & A\left(x^{2}-y^{2}-z^{2}\right)+2 B x y+2 C x z+D x+G y-F z \\
& +\theta(\psi) P+\bar{H} \\
\eta(x, y, z, \psi)= & B\left(y^{2}-x^{2}-z^{2}\right)+2 A x y+2 C y z-G x+D y+E z \\
& +\theta(\psi) Q+\bar{I} \\
\zeta(x, y, z, \psi)= & C\left(z^{2}-x^{2}-y^{2}\right)+2 A x z+2 B y z+F x-E y+D z \\
& +\theta(\psi) R+\bar{J}
\end{aligned}
$$

Четвертая же компонента определяется из уравнений (6), которые в нашем случае вьглядят как

$$
\varphi_{x}=\frac{\theta^{\prime}(\psi) P}{V^{2}(r)}, \quad \varphi_{y}=\frac{\theta^{\prime}(\psi) Q}{V^{2}(r)}, \quad \varphi_{z}=\frac{\theta^{\prime}(\psi) R}{V^{2}(r)}
$$

где $r=P x+Q y+R z$. Поскольку, очевидно, $P=r_{x}, Q=r_{y}, R=r_{z}$, из наших уравнений немедленно следует, что

$$
\varphi(x, y, z, \psi)=\theta^{\prime}(\psi) S(P x+Q y+R z)+\widehat{\varphi}(\psi), \quad S(r)=\int \frac{d r}{V^{2}(r)} .
$$


5.2. Случай функции (16). Разбирается аналогично п. 5.1. Здесь

$$
\omega(x, y, z, \psi)=\Omega\left(\sigma_{0}, \psi\right),
$$

где $\sigma_{0}=(x-\alpha(\psi))^{2}+(y-\beta(\psi))^{2}+(z-\gamma(\psi))^{2}$, условие независимости $\omega$ от $\psi$ имеет вид

$$
\begin{gathered}
-\Omega_{\sigma_{0}}\left(\sigma_{0}, \psi\right)\left(2 \alpha^{\prime}(\psi)(x-\alpha(\psi))+2 \beta^{\prime}(\psi)(y-\beta(\psi))\right. \\
\left.+2 \gamma^{\prime}(\psi)(z-\gamma(\psi))\right)+\Omega_{\psi}\left(\sigma_{0}, \psi\right) \equiv 0 .
\end{gathered}
$$

Если зафиксировать значения $\psi$ и $\sigma_{0}$, то допустимые наборы $(x, y, z)$ образуют сферу. Левая часть нашего уравнения является линейной функцией (относительно $x, y, z)$ и сохраняет на сфере нулевое значение. Это возможно, только если эта функция тривиальна:

$$
\Omega_{\sigma_{0}}\left(\sigma_{0}, \psi\right) \alpha^{\prime}(\psi)=\Omega_{\sigma_{0}}\left(\sigma_{0}, \psi\right) \beta^{\prime}(\psi)=\Omega_{\sigma_{0}}\left(\sigma_{0}, \psi\right) \gamma^{\prime}(\psi)=0,
$$

откуда в силу нетривиальности $\Omega_{\sigma_{0}}$

$$
\alpha^{\prime}(\psi)=\beta^{\prime}(\psi)=\gamma^{\prime}(\psi)=0
$$

и $\Omega_{\psi}\left(\sigma_{0}, \psi\right)=0$. Таким образом, $\omega=\Omega\left(\sigma_{0}\right), v=V\left(\sigma_{0}\right)$ и, поскольку $\alpha, \beta, \gamma$ от $\psi$ не зависят, можно считать систему координат выбранной так, что $\sigma_{0}=x^{2}+y^{2}+z^{2}$.

Поскольку при таком выборе системы координат $H^{\prime}=I^{\prime}=J^{\prime}=0$ (а остальные производные коэффициентов, кроме $D^{\prime}(\psi)$, равны нулю по предположению), первые три компоненты алгебры Ли имеют вид

$$
\begin{aligned}
\xi & =A\left(x^{2}-y^{2}-z^{2}\right)+2 B x y+2 C x z+D(\psi) x+G y-F z+H, \\
\eta & =B\left(y^{2}-x^{2}-z^{2}\right)+2 A x y+2 C y z-G x+D(\psi) y+E z+I, \\
\zeta & =C\left(z^{2}-x^{2}-y^{2}\right)+2 A x z+2 B y z+F x-E y+D(\psi) z+J .
\end{aligned}
$$

Из уравнений (6), имеющих в этом случае вид

$$
\varphi_{x}=\frac{D^{\prime}(\psi) x}{V^{2}\left(x^{2}+y^{2}+z^{2}\right)}, \quad \varphi_{y}=\frac{D^{\prime}(\psi) y}{V^{2}\left(x^{2}+y^{2}+z^{2}\right)}, \quad \varphi_{z}=\frac{D^{\prime}(\psi) z}{V^{2}\left(x^{2}+y^{2}+z^{2}\right)},
$$

получаем

$$
\varphi(x, y, z, \psi)=\frac{1}{2} D^{\prime}(\psi) S\left(x^{2}+y^{2}+z^{2}\right)+\widehat{\varphi}(\psi), \quad S(r)=\int \frac{d r}{V^{2}(r)} .
$$

5.3. Случай функции (15). Здесь нам будет удобно работать в исходной системе координат (без сдвига), обозначив $m=G^{\prime} y-F^{\prime} z+H^{\prime}, n=-G^{\prime} x+E^{\prime} z+I^{\prime}$, $r=m / n$ (по-прежнему предполагая, что $G^{\prime}(\psi) \neq 0$, и оставляя в силе условие (11) $\left.H^{\prime} E^{\prime}+I^{\prime} F^{\prime}+J^{\prime} G^{\prime}=0\right)$. Для $\omega(x, y, z, \psi)=2 \ln n+\Omega(r, \psi)$ условие независимости от $\psi$ имеет вид

$$
\Omega_{r}(r, \psi)\left(\frac{m_{\psi} n-m n_{\psi}}{n^{2}}\right)+\Omega_{\psi}(r, \psi)+2 \frac{n_{\psi}}{n}=0,
$$


и мы преобразуем его, пользуясь тем, что $m / n=r$, и введя обозначения $p=$ $m_{\psi} / m, q=n_{\psi} / n$, к виду

$$
p\left(r \Omega_{r}(r, \psi)\right)-q\left(r \Omega_{r}(r, \psi)-2\right)+\Omega_{\psi}(r, \psi)=0
$$

Покажем, что полученное равенство может выполняться только при условии $E^{\prime \prime} G^{\prime}-G^{\prime \prime} E^{\prime}=F^{\prime \prime} G^{\prime}-G^{\prime \prime} F^{\prime}=0$. Дело в том, что в предположении, что хотя бы одна из этих величин ненулевая, для любого заданного $r^{*}$ оказывается возможным считать $(p, q, r)$ новой локальной (вблизи $\left(p^{*}, q^{*}, r^{*}\right)$ для некоторых $p^{*}$ и $\left.q^{*}\right)$ криволинейной системой координат, эквивалентной $(x, y, z)$, а поэтому оказывается возможным, зафиксировав $r$, менять $p$ и $q$ произвольным образом. Но тогда из нашего уравнения получаем $r \Omega_{r}=r \Omega_{r}-2=0$, чего, очевидно, не может быть.

Эквивалентность же (локально) систем $(p, q, r)$ и $(x, y, z)$ следует из того, что $(p, q, r)$ выражаются через $(x, y, z)$ явно, а обратная зависимость определяется из системы линейных уравнений

$$
\begin{aligned}
G^{\prime} y-F^{\prime} z+H^{\prime} & =\left(-G^{\prime} x+E^{\prime} z+I^{\prime}\right) r, \\
G^{\prime \prime} y-F^{\prime \prime} z+H^{\prime \prime} & =\left(G^{\prime} y-F^{\prime} z+H^{\prime}\right) p, \\
-G^{\prime \prime} x+E^{\prime \prime} z+I^{\prime \prime} & =\left(-G^{\prime} x+E^{\prime} z+I^{\prime}\right) q,
\end{aligned}
$$

определитель которой равен $r\left(G^{\prime \prime}-p G^{\prime}\right)\left(E^{\prime \prime} G^{\prime}-G^{\prime \prime} E^{\prime}\right)+\left(G^{\prime \prime}-q G^{\prime}\right)\left(G^{\prime} F^{\prime \prime}-G^{\prime \prime} F^{\prime}\right)$. Поэтому в предположении, что хотя бы одна из величин $E^{\prime \prime} G^{\prime}-G^{\prime \prime} E^{\prime}, F^{\prime \prime} G^{\prime}-G^{\prime \prime} F^{\prime}$ не равна нулю, для любого $r^{*}$ можно указать такие $p^{*}, q^{*}$, что определитель будет отличен от нуля в окрестности соответствующей точки, а значит, в этой окрестности $(x, y, z)$ и $(p, q, r)$ взаимно однозначно выражаются друг через друга.

Таким образом, мы доказали, что условие независимости от $\psi$ может выполняться, только если $E^{\prime \prime} G^{\prime}-G^{\prime \prime} E^{\prime}=F^{\prime \prime} G^{\prime}-G^{\prime \prime} F^{\prime}=0$, что означает просто пропорциональность между собой функций $E^{\prime}, F^{\prime}, G^{\prime}: E^{\prime}(\psi)=\bar{E} \theta^{\prime}(\psi), F^{\prime}(\psi)=\bar{F} \theta^{\prime}(\psi)$, $G^{\prime}(\psi)=\bar{G} \theta^{\prime}(\psi)$, причем $\theta^{\prime}(\psi) \neq 0$. Тогда для удобства можно предполагать, что и $H^{\prime}(\psi)=\theta^{\prime}(\psi) \widehat{H}(\psi), I^{\prime}(\psi)=\theta^{\prime}(\psi) \widehat{I}(\psi), \quad J^{\prime}(\psi)=\theta^{\prime}(\psi) \widehat{J}(\psi)$. В этом случае $m=\theta^{\prime}(\psi)(\bar{G} y-\bar{F} z+\widehat{H}(\psi)), n=\theta^{\prime}(\psi)(-\bar{G} x+\bar{E} z+\widehat{I}(\psi)), r=(\bar{G} y-\bar{F} z+$ $\widehat{H}(\psi)) /(-\bar{G} x+\bar{E} z+\widehat{I}(\psi)), \omega(x, y, z, \psi)=2 \ln (-\bar{G} x+\bar{E} z+\widehat{I}(\psi))+\widehat{\Omega}(r, \psi)$. Но тогда условие независимости $\omega$ от $\psi$ упрощается и приобретает после преобразования вид

$$
\frac{1}{-\bar{G} x+\bar{E} z+\widehat{I}(\psi)}\left(\widehat{H}^{\prime}(\psi) \widehat{\Omega}_{r}(r, \psi)-\left(r \widehat{\Omega}_{r}(r, \psi)-2\right) \widehat{I}^{\prime}(\psi)\right)+\widehat{\Omega}_{\psi}(r, \psi)=0
$$

Поскольку равенство должно быть тождеством, а при зафиксированных $r, \psi$ множитель $1 /(-\bar{G} x+\bar{E} z+\widehat{I}(\psi))$ может меняться произвольным образом, полученное уравнение эквивалентно следующим двум условиям:

$$
\widehat{H}^{\prime}(\psi) \widehat{\Omega}_{r}(r, \psi)-\left(r \widehat{\Omega}_{r}(r, \psi)-2\right) \widehat{I}^{\prime}(\psi)=0, \quad \widehat{\Omega}_{\psi}(r, \psi)=0
$$


Второе условие означает, что $\widehat{\Omega}$ является функцией только одной переменной $-r$. А в первом при этом, по сушеству, происходит разделение переменных:

$$
\widehat{H}^{\prime}(\psi) \widehat{\Omega}^{\prime}(r)=\left(r \widehat{\Omega}^{\prime}(r)-2\right) \widehat{I}^{\prime}(\psi),
$$

и либо $\widehat{H}^{\prime}=\widehat{I}^{\prime}=0$, либо $r \widehat{\Omega}^{\prime}(r)-2=k \widehat{\Omega}^{\prime}(r)$ и $\widehat{H}^{\prime}=k \widehat{I}^{\prime}$.

В случае $r \widehat{\Omega}^{\prime}(r)-2=k \widehat{\Omega}^{\prime}(r)$ оказьвается $\omega=2 \ln (w(\bar{G} y-\bar{F} z+\widehat{H}(\psi))-$ $k(-\bar{G} x+\bar{E} z+\widehat{I}(\psi)))$, и мы приходим к случаю линейной функции $v(x, y, z) \mathrm{c}$ не зависящими от $\psi$ коэффициентами $(\widehat{H}(\psi)-k \widehat{I}(\psi)$ является константой, так как $\left.\widehat{H}^{\prime}(\psi)-k \widehat{I}^{\prime}(\psi)=0\right): v(x, y, z)=w(k \bar{G} x+\bar{G} y-(\bar{F}+k \bar{E}) z+M)$. Мы ее уже рассмотрели в $\S 4$, поэтому далее будем обсуждать только случай $\widehat{H}^{\prime}=\widehat{I}^{\prime}=0$, т.е. $\widehat{H}$ и $\widehat{I}$ (а значит, и $\widehat{J}$-в силу $\widehat{H} \bar{E}+\widehat{I} \bar{F}+\widehat{J} \bar{G}=0)$ являются константами: $\widehat{H}(\psi) \equiv \bar{H}$, $\widehat{I}(\psi) \equiv \bar{I}, \widehat{J}(\psi) \equiv \bar{J}$. Но тогда мы приходим к функции скорости

$$
v(x, y, z)=(-\bar{G} x-\bar{E} z+\bar{I}) V\left(\frac{\bar{G} y-\bar{F} z+\bar{H}}{-\bar{G} x+\bar{E} z+\bar{I}}\right)
$$

причем $\bar{E} \bar{H}+\bar{F} \bar{I}+\bar{G} \bar{J}=0$. Далее мы будем предполагать, что за счет сдвига начала координат $\bar{H}, \bar{I}$ и $\bar{J}$ сделаны нулевыми, и тогда (черточки над $E, F, G$ мы далее будем опускать)

$$
v(x, y, z)=(-G x+E z) V\left(\frac{G y-F z}{-G x+E z}\right),
$$

а первые три компоненты алгебры Ли имеют вид

$$
\begin{aligned}
\xi= & A\left(x^{2}-y^{2}-z^{2}\right)+2 B x y+2 C x z+D x+\theta(\psi)(G y-F z) \\
& +G_{1} y-F_{1} z+H_{1} \\
\eta= & B\left(y^{2}-x^{2}-z^{2}\right)+2 A x y+2 C y z+D y+\theta(\psi)(-G x+E z) \\
& -G_{1} x+E_{1} z+I_{1} \\
\zeta= & C\left(z^{2}-x^{2}-y^{2}\right)+2 A x z+2 B y z+D z+\theta(\psi)(F x-E y) \\
& +F_{1} x-E_{1} y+J_{1} .
\end{aligned}
$$

При этом мы для однозначности можем считать, что вектор $(E, F, G)$ нормирован: $E^{2}+F^{2}+G^{2}=1$, и что $E E_{1}+F F_{1}+G G_{1}=0$ (иначе можно составляющую вектора $\left(E_{1}, F_{1}, G_{1}\right)$, коллинеарную $(E, F, G)$, просто включить в предыдушее слагаемое, заменив $\theta(\psi)+E E_{1}+F F_{1}+G G_{1}$ на $\left.\widehat{\theta}(\psi)\right)$.

Четвертая же компонента $\varphi$ определяется, как и раншше, из соотношений (6), которые в нашем случае имеют вид

$$
\varphi_{x}=\theta^{\prime}(\psi) \frac{G y-F z}{V^{2}(r)(-G x+E z)^{2}}=\frac{\theta^{\prime}(\psi)}{G V^{2}(r)} r_{x}, \quad \varphi_{y}=\frac{\theta^{\prime}(\psi)}{G V^{2}(r)} r_{y}, \quad \varphi_{z}=\frac{\theta^{\prime}(\psi)}{G V^{2}(r)} r_{z}
$$

(через $r$ мы, по-прежнему, обозначаем дробь $r=(G y-F z) /(-G x+E z))$, откуда, очевидно,

$$
\varphi(x, y, z, \psi)=\frac{\theta^{\prime}(\psi)}{G} S(r)+\widehat{\varphi}(\psi), \quad S(r)=\int \frac{d r}{V^{2}(r)} .
$$


5.4. Случай функции (13). Обозначая, как и ранее, $\widetilde{x}=x-\alpha(\psi), \widetilde{y}=$ $y-\beta(\psi), \widetilde{z}=z-\gamma(\psi), l=A^{\prime} \widetilde{x}+B^{\prime} \widetilde{y}+C^{\prime} \widetilde{z}, \sigma=\widetilde{x}^{2}+\widetilde{y}^{2}+\widetilde{z}^{2} \pm \nu^{2}, r=l / \sigma$, выпишем условия независимости от $\psi$ функции $\omega(x, y, z, \psi)=2 \ln \sigma+\Omega(r, \psi)$. После преобразования они приобретают вид

$$
\begin{aligned}
& -2\left(\alpha^{\prime}(x-\alpha)+\beta^{\prime}(y-\beta)+\gamma^{\prime}(z-\gamma) \mp \nu \nu^{\prime}\right) r\left(2-r \Omega_{r}(r, \psi)\right) \\
& \quad+r \Omega_{r}(r, \psi)\left(A^{\prime \prime}(x-\alpha)+B^{\prime \prime}(y-\beta)+C^{\prime \prime}(z-\gamma)-\left(A^{\prime} \alpha^{\prime}+B^{\prime} \beta^{\prime}+C^{\prime} \gamma^{\prime}\right)\right) \\
& \quad+\Omega_{\psi}(r, \psi)\left(A^{\prime}(x-\alpha)+B^{\prime}(y-\beta)+C^{\prime}(z-\gamma)\right)=0 .
\end{aligned}
$$

Если зафиксировать $r, \psi$, то полученное соотношение означает равенство нулю линейной относительно $(x, y, z)$ функции на сфере

$$
(x-\alpha)^{2}+(y-\beta)^{2}+\left(z-\gamma^{2}\right) \pm \nu^{2}-\frac{1}{r}\left(A^{\prime}(x-\alpha)+B^{\prime}(y-\beta)+C^{\prime}(z-\gamma)\right)=0,
$$

что возможно, только если все ее коэффициенты нулевые. Это дает нам систему уравнений

$$
\begin{aligned}
-2 \alpha^{\prime} r\left(2-r \Omega_{r}(r, \psi)\right)+r \Omega_{r}(r, \psi) A^{\prime \prime}+\Omega_{\psi}(r, \psi) A^{\prime} & =0, \\
-2 \beta^{\prime} r\left(2-r \Omega_{r}(r, \psi)\right)+r \Omega_{r}(r, \psi) B^{\prime \prime}+\Omega_{\psi}(r, \psi) B^{\prime} & =0, \\
-2 \gamma^{\prime} r\left(2-r \Omega_{r}(r, \psi)\right)+r \Omega_{r}(r, \psi) C^{\prime \prime}+\Omega_{\psi}(r, \psi) C^{\prime} & =0, \\
\pm 2 \nu \nu^{\prime} r\left(2-r \Omega_{r}(r, \psi)\right)-r \Omega_{r}(r, \psi)\left(A^{\prime} \alpha^{\prime}+B^{\prime} \beta^{\prime}+C^{\prime} \gamma^{\prime}\right) & =0 .
\end{aligned}
$$

Исключая пропорциональность $r\left(2-r \Omega_{r}\right)$ и $r \Omega_{r}$ (так как предположение, что $r\left(2-r \Omega_{r}(r, \psi)\right)=k(\psi) r \Omega_{r}(r, \psi)$, приводит к $v(x, y, z)=\Delta(\psi)(l+k \sigma)$, которая является линейной функцией при $k=0$ и квадратичной при $k \neq 0$ ), из последнего уравнения получаем $\nu^{\prime}=0$ и $A^{\prime} \alpha^{\prime}+B^{\prime} \beta^{\prime}+C^{\prime} \gamma^{\prime}=0$. Сложением первого уравнения, умноженного на $\alpha^{\prime}$, второго - на $\beta^{\prime}$ и третьего - на $\gamma^{\prime}$ получаем

$$
-2\left(\alpha^{2}+\beta^{\prime 2}+\gamma^{\prime 2}\right) r\left(2-r \Omega_{r}(r, \psi)\right)+r \Omega_{r}(r, \psi)\left(A^{\prime \prime} \alpha^{\prime}+B^{\prime \prime} \beta^{\prime}+C^{\prime \prime} \gamma^{\prime}\right)=0,
$$

откуда (опять же исключая пропорциональность $r\left(2-r \Omega_{r}\right)$ и $\left.r \Omega_{r}\right) \alpha^{\prime}=\beta^{\prime}=$ $\gamma^{\prime}=0$, т.е. $\sigma$ от $\psi$ не зависит. Нам будет удобно далее считать, что за счет сдвига системы координат мы добились того, чтобы $\alpha=\beta=\gamma=0$, т.е. $\sigma=x^{2}+y^{2}+$ $z^{2} \pm \nu^{2}$, a $l=A^{\prime} x+B^{\prime} y+C^{\prime} z$.

В указанных предположениях наши уравнения приобретают вид

$$
\begin{aligned}
& r \Omega_{r}(r, \psi) A^{\prime \prime}+\Omega_{\psi}(r, \psi) A^{\prime}=r \Omega_{r}(r, \psi) B^{\prime \prime}+\Omega_{\psi}(r, \psi) B^{\prime} \\
& \quad=r \Omega_{r}(r, \psi) C^{\prime \prime}+\Omega_{\psi}(r, \psi) C^{\prime}=0
\end{aligned}
$$

что означает взаимную пропорциональность коэффициентов $A^{\prime}, B^{\prime}, C^{\prime}: A^{\prime}(\psi)=$ $\Delta^{\prime}(\psi) P, B^{\prime}(\psi)=\Delta^{\prime}(\psi) Q, C^{\prime}(\psi)=\Delta^{\prime}(\psi) R$, причем мы можем предполагать для определенности $P^{2}+Q^{2}+R^{2}=1$. В этом случае $l=A^{\prime} x+B^{\prime} y+C^{\prime} z=\Delta^{\prime}(\psi)(P x+$ $Q y+R z)$,

$$
\omega=2 \ln \left(x^{2}+y^{2}+z^{2} \pm \nu^{2}\right)+\widehat{\Omega}\left(\frac{P x+Q y+R z}{x^{2}+y^{2}+z^{2} \pm \nu^{2}}, \psi\right)
$$


и независимость $\omega$ от $\psi$ эквивалентна $\widehat{\Omega}_{\psi}=0$, так что окончательно получаем

$$
v(x, y, z)=\sigma V(r), \quad r=\frac{l}{\sigma}=\frac{P x+Q y+R z}{x^{2}+y^{2}+z^{2} \pm \nu^{2}} .
$$

Первые три компоненты соответствуюшей алгебры Ли имеют вид

$$
\begin{aligned}
& \xi(x, y, z, \psi)=\Delta(\psi)\left(P\left(x^{2}-y^{2}-z^{2} \mp \nu^{2}\right)+2 Q x y+2 R x z\right) \\
& \quad+\bar{A}\left(x^{2}-y^{2}-z^{2} \mp \nu^{2}\right)+2 \bar{B} x y+2 \bar{C} x z+D x+G y-F z+H \\
& \eta(x, y, z, \psi)=\Delta(\psi)\left(Q\left(y^{2}-x^{2}-z^{2} \mp \nu^{2}\right)+2 P x y+2 R y z\right) \\
& \quad+\bar{B}\left(y^{2}-x^{2}-z^{2} \mp \nu^{2}\right)+2 \bar{A} x y+2 \bar{C} y z-G x+D y+E z+I \\
& \zeta(x, y, z, \psi)=\Delta(\psi)\left(R\left(z^{2}-y^{2}-x^{2} \mp \nu^{2}\right)+2 Q y z+2 P x z\right) \\
& \quad+\bar{C}\left(z^{2}-y^{2}-x^{2} \mp \nu^{2}\right)+2 \bar{B} y z+2 \bar{A} x z+F x-E y+D z+J
\end{aligned}
$$

причем без ограничения общности можно считать $\bar{A} P+\bar{B} Q+\bar{C} R=0$.

Четвертая же компонента опять легко определяется из уравнений (6): поскольку оказывается, что $\xi_{\psi}=-\Delta^{\prime}(\psi) r_{x} \sigma^{2}, \eta_{\psi}=-\Delta^{\prime}(\psi) r_{y} \sigma^{2}, \quad \zeta_{\psi}=-\Delta^{\prime}(\psi) r_{z} \sigma^{2}$, уравнения

$$
\varphi_{x}=\frac{\xi_{\psi}}{v^{2}}, \quad \varphi_{y}=\frac{\eta_{\psi}}{v^{2}}, \quad \varphi_{z}=\frac{\zeta_{\psi}}{v^{2}}
$$

немедленно дают

$$
\varphi(x, y, z, \psi)=-\Delta^{\prime}(\psi) S(r)+\widehat{\varphi}(\psi), \quad S(r)=\int \frac{d r}{V^{2}(r)} .
$$

5.5. Случай п. 3.8. Здесь на вид функций $\omega$ и $v$ пока никаких ограничений не налагалось, поэтому независимость их от $\psi$ никаких дополнительных предположений не требует, а система (6) преврашается в $\varphi_{x}=\varphi_{y}=\varphi_{z}=0$, что означает, что $\varphi$ зависит только от одного аргумента: $\varphi(x, y, z, \psi)=\widehat{\varphi}(\psi)$. Остальные же компоненты алгебры определяются формулами $(7)$ с не зависящими от $\psi$ коэффициентами $A, B, C, D, E, F, G, H, I, J$.

\section{§6. Детальная групповая классификация для семейств (II.1)-(II.4)}

6.1. Последнее уравнение. По существу, у нас осталось одно-единственное уравнение, которое мы пока не разрешили - это последнее из уравнений (4)

$$
\xi_{x}=\varphi_{\psi}+\frac{1}{v}\left(\xi v_{x}+\eta v_{y}+\zeta v_{z}\right)
$$

Мы произведем подстановку в это уравнение найденных нами компонент алгебры Ли $\xi, \eta, \zeta, \varphi$ и функции скорости $v(x, y, z)$ и найдем дополнительные ограничения на коэффициенты алгебры и либо дополнительные ограничения на $v(x, y, z)($ и $S(r))$, либо найдем алгебры, обслуживающие все семейство.

Всюду рассуждения происходят в три этапа. На первом подстановкой в (27) и исключением случаев, описываемых формулами (I.1)-(I.5), удается добиться 
практически полного определения коэффициентов в формулах первых трех компонент $\xi, \eta, \zeta$ векторного поля и прийти к некоторому уравнению, в котором фигурируют уже только две независимые переменные - $r$ и $\psi$ (и в которое входят неизвестные пока функции $S(r)$ и $V(r))$. Это уравнение мы будем называть характеристическим уравнением семейства. Второй этап - это разделение переменных. Здесь “отделяется" случай алгебры Ли, обслуживающей все семейство (и тогда полученное нами уравнение удовлетворяется тождественно), а в случае семейства (II.1) (плоских функций скорости) - и случай степенной зависимости с пятимерной алгеброй Ли. Альтернативой отделившимся вариантам оказывается дифференциальное уравнение, в котором фигурирует только одна независимая переменная $-r$ и в котором задается связь между $S(r)$ и $V(r)$. Это уравнение мы будем далее называть уравнением ветвления. Третий этап - это решение уравнения ветвления. Поскольку оно имеет практически одинаковый вид для всех четырех семейств, мы соответствуюшие рассуждения проведем в общем виде в п. 6.6. Именно это уравнение дает шесть различных формул в зависимости от значений коэффициентов, и именно эти шесть вариантов и приведены в таблицах 3-7. В случае четвертого семейства возникает еще одно ветвление - в зависимости от значения выражения $\pm \nu^{2}$, так что вариантов здесь оказывается восемнадцать. Правда, некоторые из них (два варианта в случае семейства плоских функций и два - в случае осесимметричных) оказьваются функциями вида (I.1)-(I.5), и поэтому они в числе подсемейств раздела II не учитываются. Поскольку выкладки здесь достаточно громоздкие, мы их будем приводить с сушественными сокрашениями, подробно исследование всех вариантов изложено в [16].

6.2. Случай $v(x, y, z)=V(r)=V(P x+Q y+R z)$. Для такой функции $v$ (и соответствующей $\varphi$ ) уравнение $(27)$ преврашается в

$$
\xi_{x}=\theta^{\prime \prime}(\psi) S(r)+\widehat{\varphi}^{\prime}(\psi)+\frac{V^{\prime}(r)}{V(r)}\left(\xi r_{x}+\eta r_{y}+\zeta r_{z}\right),
$$

а подстановка в него $\xi, \eta, \zeta$ из (19) дает

$$
\begin{aligned}
2 A x & +2 B y+2 C z+D=\theta^{\prime \prime}(\psi) S(r)+\widehat{\varphi}^{\prime}(\psi) \\
& +\frac{V^{\prime}(r)}{V(r)}\left(P\left(A\left(x^{2}-y^{2}-z^{2}\right)+2 B x y+2 C x z+D x+E y-F z+\theta(\psi) P+\bar{H}\right)\right. \\
& +Q\left(B\left(y^{2}-x^{2}-z^{2}\right)+2 A x y+2 C y z-E x+D y+G z+\theta(\psi) Q+\bar{I}\right) \\
& \left.+R\left(C\left(z^{2}-x^{2}-y^{2}\right)+2 A x z+2 B y z+F x-G y+D z+\theta(\psi) R+\bar{J}\right)\right) .
\end{aligned}
$$

Если зафиксировать $\psi$ и $r$, то мы получим, что некоторая квадратичная функция равна нулю на гиперплоскости $P x+Q y+R z=r$, что возможно, только если эта квадратичная функция является произведением $P x+Q y+R z-r$ на некоторую другую линейную функцию от $x, y, z$, т.е. может быть представлена в виде

$$
(P x+Q y+R z-r)(\alpha x+\beta y+\gamma z-\varkappa) .
$$

Осуществляя перемножение в этой формуле и уравнивая коэффициенты при соответствуюших степенях $x, y, z$ с коэффициентами в нашем уравнении, получаем 


$$
\begin{aligned}
& \left\{\begin{array}{l}
\frac{V^{\prime}(r)}{V(r)}(A P-B Q-R C)=\alpha P \\
\frac{V^{\prime}(r)}{V(r)}(-A P+B Q-R C)=\beta Q \\
\frac{V^{\prime}(r)}{V(r)}(-A P-B Q+R C)=\gamma R
\end{array}\right. \\
& \left\{\begin{array}{l}
\frac{V^{\prime}(r)}{V(r)}(2 B P+2 A Q)=\alpha Q+\beta P ; \\
\frac{V^{\prime}(r)}{V(r)}(2 C P+2 A R)=\alpha R+\gamma P ; \\
\frac{V^{\prime}(r)}{V(r)}(2 C Q+2 B R)=\beta R+\gamma Q
\end{array}\right. \\
& \left\{\begin{array}{l}
\frac{V^{\prime}(r)}{V(r)}(P D-Q E+R F)-2 A=-\alpha r-\varkappa P ; \\
\frac{V^{\prime}(r)}{V(r)}(P E+Q D-R G)-2 B=-\beta r-\varkappa Q ; \\
\frac{V^{\prime}(r)}{V(r)}(-P F+Q G+R D)-2 C=-\gamma r-\varkappa R
\end{array}\right. \\
& \theta^{\prime \prime}(\psi) S(r)+\widehat{\varphi}^{\prime}(\psi)+\frac{V^{\prime}(r)}{V(r)}\left(\theta(\psi)\left(P^{2}+Q^{2}+R^{2}\right)+P \bar{H}+Q \bar{I}+R \bar{J}\right)-D=r \varkappa .
\end{aligned}
$$

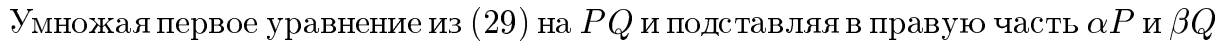
из (28), после сокрашения на $V^{\prime} / V$ (случай $V^{\prime}=0$ исключен - это случай функции (I.1)) получаем $(A P+B Q+C R)\left(P^{2}+Q^{2}\right)=0$. Аналогично выводятся равенства $(A P+B Q+C R)\left(P^{2}+R^{2}\right)=0,(A P+B Q+C R)\left(Q^{2}+R^{2}\right)=0$, откуда (в силу $P^{2}+Q^{2}+R^{2}=1$ ) следует $A P+B Q+C R=0$. Но тогда (28) и (29) дают явные выражения $\alpha, \beta, \gamma$ через $A, B, C: \alpha=2 A V^{\prime}(r) / V(r), \beta=2 B V^{\prime}(r) / V(r)$, $\gamma=2 C V^{\prime}(r) / V(r)$, и при этом из $A P+B Q+C R=0$ немедленно следует $\alpha P+\beta Q+\gamma R=0$.

Складывая уравнения (30) с коэффициентами $P, Q, R$, получаем явное выражение для $\varkappa=-D V^{\prime}(r) / V(r)$, и поэтому (30) сводится к

$$
\begin{aligned}
\frac{V^{\prime}(r)}{V(r)}(-Q E+R F) & =2 A\left(1-r \frac{V^{\prime}(r)}{V(r)}\right), \\
\frac{V^{\prime}(r)}{V(r)}(P E-R G) & =2 B\left(1-r \frac{V^{\prime}(r)}{V(r)}\right), \\
\frac{V^{\prime}(r)}{V(r)}(-P F+Q G) & =2 C\left(1-r \frac{V^{\prime}(r)}{V(r)}\right) .
\end{aligned}
$$

Отсюда исключением случая $\left(1-r V^{\prime}(r) / V(r)\right)=k V^{\prime}(r) / V(r)$ (приводящего к случаю линейной функции скорости $V(r)=w \cdot(r+k))$ получаем $A=B=$ $C=\alpha=\beta=\gamma=0,-Q E+R F=P E-R G=-P F+Q G=0$, а последние соотношения означают, что для некоторой постоянной $\Delta$ выполнено $E=\Delta R$, 
$F=\Delta Q, G=\Delta P$. Подстановка найденных коэффициентов в (19) дает

$$
\begin{aligned}
& \xi(x, y, z, \psi)=D x+\Delta(R y-Q z)+\theta(\psi) P+\bar{H}, \\
& \eta(x, y, z, \psi)=D y+\Delta(-R x+P z)+\theta(\psi) Q+\bar{I} \\
& \zeta(x, y, z, \psi)=D z+\Delta(Q x-P y)+\theta(\psi) R+\bar{J},
\end{aligned}
$$

что с учетом введенных нами в п. 1.2 обозначений приводит к алгебре Ли

$$
\begin{aligned}
\Xi_{\mathrm{II} .1}= & D \Xi_{*}+\Delta \Xi_{\circ}(P, Q, R)+\Xi_{\uparrow}(H, I, J)+\theta(\psi) \Xi_{\uparrow}(P, Q, R) \\
& +\left(\theta^{\prime}(\psi) S(P x+Q y+R z)+\widehat{\varphi}(\psi)\right) \partial_{\psi},
\end{aligned}
$$

где константы $\Delta, \bar{H}, \bar{I}, \bar{J}$ произвольны и подчиняются только ограничению $P \bar{H}+$ $Q \bar{I}+R \bar{J}=0$, а функции $S(r), V(r), \theta(\psi)$ и $\widehat{\varphi}(\psi)$ удовлетворяют уравнению

$$
\theta^{\prime \prime}(\psi) S(r)+\hat{\varphi}^{\prime}(\psi)+\frac{V^{\prime}(r)}{V(r)} \theta(\psi)=D\left(1-r \frac{V^{\prime}(r)}{V(r)}\right),
$$

которое получается из (31) подстановкой найденного значения $\varkappa$ и использованием равенства $P \bar{H}+Q \bar{I}+R \bar{J}=0$. Это и есть характеристическое уравнение первого семейства.

Разделение переменных осуществляется непосредственно: случай $\theta(\psi)=0$ при $D=0$ дает $\widehat{\varphi}=L$ и мы получаем алгебру, обслуживающую все семейство (она приведена в первой строке таблицы 2). Случай $D \neq 0$ для $\theta(\psi)=0$ (а также и для $\theta(\psi)=\bar{\theta}=\mathrm{const})$ приводит к $V(r)=w \cdot(D r+\bar{\theta})^{1-M / D}$, откуда, обозначая $-M / D=k$ и сдвигая систему координат так, чтобы $\bar{\theta}$ оказалось равным нулю, получаем подсемейство, приведенное в первой строке таблицы 3.

Если же $\theta \not \equiv$ const, то дифференцирование нашего уравнения по $\psi$ немедленно приводит к тому, что

$$
\frac{V^{\prime}(r)}{V(r)}=\lambda S(r)+\mu
$$

для некоторых констант $\lambda$ и $\mu$, подстановка этого соотношения в (33) дает

$$
S(r)\left(\theta^{\prime \prime}(\psi)+\lambda \theta(\psi)+\lambda D r\right)+\widehat{\varphi}^{\prime}(\psi)+\mu \theta(\psi)-D(1-\mu r)=0,
$$

откуда либо $\lambda D=\mu D=0, \theta^{\prime \prime}(\psi)+\lambda \theta(\psi)=0, \widehat{\varphi}^{\prime}(\psi)+\mu \theta(\psi)=D$, либо мы приходим к одной из функций скорости (I.1), (I.2). Поскольку $\lambda=\mu=0$ в силу (34) опять же приводит к функции вида (I.1), у нас остается $D=0$ и уравнение (34) дополняется уравнениями

$$
\theta^{\prime \prime}(\psi)+\lambda \theta(\psi)=0, \quad \widehat{\varphi}^{\prime}(\psi)+\mu \theta(\psi)=0
$$

По существу, разделение переменных сделано. Уравнение (34) есть уравнение ветвления для первого семейства. Здесь мы на время остановимся, поскольку анализ систем типа (34), (35) (а такие системы возникают во всех четырех случаях) мы будем проводить единым образом в п. 6.6 . 
6.3. Случай $v(x, y, z)=V\left(\sigma_{0}\right)=V\left(x^{2}+y^{2}+z^{2}\right)$. Анализ этого случая происходит по той же схеме, что и в п. 6.2. Подстановка в уравнение $(27) \xi, \eta$, $\zeta, \varphi$ из (21), (22) и $v$ из (II.2) дает линейное уравнение относительно $x, y, z$ (при зафиксированных $\psi$ и $\sigma_{0}$ ), которое оказывается тождеством на сфере $x^{2}+y^{2}+$ $z^{2}=\sigma_{0}$. Это возможно, только если линейные функции в правой и левой частях уравнения совпадают тождественно, что дает систему соотношений

$$
\begin{gathered}
2 A=2 \frac{V^{\prime}\left(\sigma_{0}\right)}{V\left(\sigma_{0}\right)}\left(A \sigma_{0}+H\right), \quad 2 B=2 \frac{V^{\prime}\left(\sigma_{0}\right)}{V\left(\sigma_{0}\right)}\left(B \sigma_{0}+I\right), \quad 2 C=2 \frac{V^{\prime}\left(\sigma_{0}\right)}{V\left(\sigma_{0}\right)}\left(C \sigma_{0}+J\right), \\
\frac{1}{2} D^{\prime \prime}(\psi) S\left(\sigma_{0}\right)+\hat{\varphi}^{\prime}(\psi)+D(\psi)\left(2 \frac{V^{\prime}\left(\sigma_{0}\right)}{V\left(\sigma_{0}\right)} \sigma_{0}-1\right)=0
\end{gathered}
$$

из которой исключением случая функции скорости вида (І.5) получаем $A=B=$ $C=H=I=J=0$, так что нам остается единственное уравнение (36) - характеристическое уравнение второго семейства. Компоненты векторного поля $\xi, \eta, \zeta$ приобретают вид

$$
\xi=D(\psi) x+G y-F z, \quad \eta=-G x+D(\psi) y+E z, \quad \zeta=F x-E y+D(\psi) z,
$$

что приводит с учетом обозначений п. 1.2 к алгебре

$$
\Xi_{\mathrm{II} .2}=\Xi_{\circ}(E, F, G)+D(\psi) \Xi_{*}+\left(\frac{1}{2} D^{\prime}(\psi) S\left(x^{2}+y^{2}+z^{2}\right)+\widehat{\varphi}(\psi)\right) \partial_{\psi}
$$

с произвольньми константами $E, F, G$ и функциями $S(r), V(r), D(\psi), \widehat{\varphi}(\psi)$, удовлетворяющими (36).

Разделение переменных в (36) дает либо $D(\psi) \equiv 0$, что приводит к $\varphi(\psi)=L$, и мы получаем алгебру, обслуживающую все семейство, которая приведена во второй строке таблицы 2 . Либо $D(\psi) \neq 0$ (хотя бы для одного значения $\psi$ ), и мы получаем тогда уравнение ветвления

$$
4 \frac{V^{\prime}\left(\sigma_{0}\right)}{V\left(\sigma_{0}\right)} \sigma_{0}-2=\lambda S+\mu
$$

а из (36) - сопровождающие уравнения

$$
D^{\prime \prime}(\psi)+\lambda D(\psi)=0, \quad \hat{\varphi}^{\prime}(\psi)+\frac{1}{2} \mu D(\psi)=0 .
$$

Это - система того же типа, что и (34), (35), она будет обсуждаться в п. 6.6 .

6.4. Случай $v(x, y, z)=(-G x+E z) V(r)=(-G x+E z) V((G y-F z) /(-G x+$ $E z))$. Подстановка в уравнение $(27) \xi, \eta, \zeta, \varphi$ из (23), (24) и $v$ дает квадратичное уравнение относительно $x, y, z$ (при зафиксированных $\psi$ и $r$ ), которое оказывается тождеством на плоскости $r=(G y-F z) /(-G x+E z)$. Поскольку гомотетия с коэффициентом $k$ оставляет нас на этой плоскости, а различные члены квадратичной функции при этом умножаются на разные коэффициенты, оказывается возможным 
приравнять друг другу по отдельности квадратичные, линейные и свободные члены, что дает три уравнения

$$
\begin{aligned}
& (G A-E C)\left(1-r \frac{V^{\prime}(r)}{V(r)}\right)-(G B-F C) \frac{V^{\prime}(r)}{V(r)}=0 \\
& (-G x+E z)\left(\frac{1}{G} \theta^{\prime \prime}(\psi) S(r)+\widehat{\varphi}^{\prime}(\psi)\right)-G\left(\theta(\psi)(G y-F z)+G_{1} y-F_{1} z\right) \\
& \quad+E\left(\theta(\psi)(F x-E y)+F_{1} x-E_{1} y\right)+\frac{V^{\prime}(r)}{V(r)}\left(G r\left(\theta(\psi)(G y-F z)+G_{1} y-F_{1} z\right)\right. \\
& \quad+G\left(\theta(\psi)(-G x+E z)-G_{1} x+E_{1} z\right) \\
& \left.\quad-(F+E r)\left(\theta(\psi)(F x-E y)+F_{1} x-E_{1} y\right)\right)=0, \\
& \left(E J_{1}-G H_{1}\right)\left(1-r \frac{V^{\prime}(r)}{V(r)}\right)-\frac{V^{\prime}(r)}{V(r)}\left(F J_{1}-G I_{1}\right)=0 .
\end{aligned}
$$

Исключение в первом и третьем уравнениях случая функции вида (I.2) приводит нас к условиям $G A-C E=G B-F C=0, E J_{1}-G H_{1}=F J_{1}-G I_{1}=0$, второе уравнение приводится к виду

$$
\begin{aligned}
(-G x & +E z)\left(\frac{1}{G} \theta^{\prime \prime}(\psi) S(r)+\widehat{\varphi}^{\prime}(\psi)\right. \\
& \left.+G \theta(\psi)\left(-r-\frac{E(F+E r)}{G^{2}}+\frac{V^{\prime}(r)}{V(r)}\left(r^{2}+1+\frac{(F+E r)^{2}}{G^{2}}\right)\right)\right) \\
= & \left(-F_{1}+\left(F_{1} r-E_{1}\right) \frac{V^{\prime}(r)}{V(r)}\right)(E x+F y+G z),
\end{aligned}
$$

и подстановка в это уравнение вектора $(x, y, z)=(E-\varepsilon, F+\varepsilon r, G)$ (очевидно, лежащего в плоскости $r=(G y-F z) /(-G x+E z))$ дает в силу произвольности выбора $\varepsilon$ два уравнения, первое из которых $-F_{1}+\left(F_{1} r-E_{1}\right) V^{\prime}(r) / V(r)=0$ приводит исключением случая функций вида (I.1), (I.2) к условию $E_{1}=F_{1}=G_{1}=0$, а второе тогда оказывается как раз характеристическим уравнением третьего семейства:

$\frac{1}{G} \theta^{\prime \prime}(\psi) S(r)+\widehat{\varphi}^{\prime}(\psi)+G \theta(\psi)\left(-r-\frac{E(F+E r)}{G^{2}}+\frac{V^{\prime}(r)}{V(r)}\left(r^{2}+1+\frac{(F+E r)^{2}}{G^{2}}\right)\right)=0$.

Подстановка полученных нами значений коэффициентов в (23) дает

$$
\begin{aligned}
& \xi(x, y, z, \psi)=\Delta\left(E\left(x^{2}-y^{2}-z^{2}\right)+2 F x y+2 G x z\right)+D x+\theta(\psi)(E y-F z)+\varkappa E, \\
& \eta(x, y, z, \psi)=\Delta\left(F\left(y^{2}-x^{2}-z^{2}\right)+2 E x y+2 G y z\right)+D y+\theta(\psi)(-E x+G z)+\varkappa F, \\
& \zeta(x, y, z, \psi)=\Delta\left(G\left(z^{2}-x^{2}-y^{2}\right)+2 E x z+2 F y z\right)+D z+\theta(\psi)(F x-G y)+\varkappa G,
\end{aligned}
$$

и мы получаем алгебру Ли

$$
\begin{aligned}
\Xi_{\mathrm{II} .3}= & \Delta \Xi_{\infty}(E, F, G, 0)+D \Xi_{*}+\varkappa \Xi_{\uparrow}(E, F, G)+\theta(\psi) \Xi_{\circ}(E, F, G) \\
& +\left(\frac{\theta^{\prime}(\psi)}{G} S\left(\frac{G y-F z}{-G x+E z}\right)+\widehat{\varphi}(\psi)\right) \partial_{\psi}
\end{aligned}
$$


с произвольными константами $\Delta, D, \varkappa$ и с функциями $S(r), V(r), \theta(\psi)$ и $\widehat{\varphi}(\psi)$, удовлетворяюшими (40).

Из (40) при $\theta(\psi) \equiv 0$ мы получаем $\varphi(\psi)=L$ и приходим к алгебре, обслуживающей все семейство, которая указана в третьей строке таблицы 2 . При $\theta(\psi) \neq 0$ хотя бы для одного $\psi$ мы получаем, как и в предыдущих пунктах, систему

$$
\begin{gathered}
\frac{V^{\prime}(r)}{V(r)}\left(r^{2}+1+\frac{(F+E r)^{2}}{G^{2}}\right)-r-\frac{E(F+E r)}{G^{2}}=\frac{1}{G}(\lambda S(r)+\mu), \\
\theta^{\prime \prime}(\psi)+\lambda G \theta(\psi)=0, \quad \hat{\varphi}^{\prime}(\psi)=-\mu \theta(\psi),
\end{gathered}
$$

к которой вернемся в п. 6.6 .

6.5. Случай $v(x, y, z)=\left(x^{2}+y^{2}+z^{2} \pm \nu^{2}\right) V\left((P x+Q y+R z) /\left(x^{2}+y^{2}+z^{2} \pm\right.\right.$ $\left.\left.\nu^{2}\right)\right)$. Подстановка в уравнение (27) $\xi, \eta, \zeta, \varphi$ и $v$ из $(25),(26)$ дает после упрошений и фиксации, как и ранее, $\psi$ и $r=(P x+Q y+R z) /\left(x^{2}+y^{2}+z^{2} \pm \nu^{2}\right)$ равенство нулю линейной функции на сфере. Это возможно, только если линейная функция тривиальна, что дает систему соотношений

$$
\begin{gathered}
P\left(-\Delta^{\prime \prime}(\psi) S(r)+\widehat{\varphi}^{\prime}(\psi)+D\left(1-r \frac{V^{\prime}(r)}{V(r)}\right)-\Delta(\psi)\left(\frac{V^{\prime}(r)}{V(r)}\left(1 \mp 4 r^{2} \nu^{2}\right) \pm 4 r \nu^{2}\right)\right) \\
\mp 4 \bar{A}\left(r-\frac{V^{\prime}(r)}{V(r)} r^{2}\right) \nu^{2}+2 H\left(r-r^{2} \frac{V^{\prime}(r)}{V(r)}\right)+(F R-G Q) r \frac{V^{\prime}(r)}{V(r)}=0, \\
Q\left(-\Delta^{\prime \prime}(\psi) S(r)+\widehat{\varphi}^{\prime}(\psi)+D\left(1-r \frac{V^{\prime}(r)}{V(r)}\right)-\Delta(\psi)\left(\frac{V^{\prime}(r)}{V(r)}\left(1 \mp 4 r^{2} \nu^{2}\right) \pm 4 r \nu^{2}\right)\right) \\
\mp 4 \bar{B}\left(r-\frac{V^{\prime}(r)}{V(r)} r^{2}\right) \nu^{2}+2 I\left(r-r^{2} \frac{V^{\prime}(r)}{V(r)}\right)+(G P-E R) r \frac{V^{\prime}(r)}{V(r)}=0, \\
R\left(-\Delta^{\prime \prime}(\psi) S(r)+\widehat{\varphi}^{\prime}(\psi)+D\left(1-r \frac{V^{\prime}(r)}{V(r)}\right)-\Delta(\psi)\left(\frac{V^{\prime}(r)}{V(r)}\left(1 \mp 4 r^{2} \nu^{2}\right) \pm 4 r \nu^{2}\right)\right) \\
\mp 4 \bar{C}\left(r-\frac{V^{\prime}(r)}{V(r)} r^{2}\right) \nu^{2}+2 J\left(r-r^{2} \frac{V^{\prime}(r)}{V(r)}\right)+(E Q-F P) r \frac{V^{\prime}(r)}{V(r)}=0, \\
r \frac{V^{\prime}(r)}{V(r)}\left( \pm 2 D r \nu^{2}+(P H+Q I+R J)\right) \mp 2 D r \nu^{2}=0 .
\end{gathered}
$$

Исключение в последнем соотношении случаев функций вида (I.2)-(I.5) приводит к равенствам $D=0, P H+Q I+R J=0$, а тогда из первых трех соотношений получаем четыре уравнения:

$$
\begin{gathered}
-\Delta^{\prime \prime}(\psi) S(r)+\widehat{\varphi}^{\prime}(\psi)-\Delta(\psi)\left(\frac{V^{\prime}(r)}{V(r)}\left(1 \mp 4 r^{2} \nu^{2}\right) \pm 4 r \nu^{2}\right)=0, \\
\left(2 H \mp 4 \bar{A} \nu^{2}\right)\left(r-\frac{V^{\prime}(r)}{V(r)} r^{2}\right)+(F R-G Q) r \frac{V^{\prime}(r)}{V(r)}=0, \\
\left(2 I \mp 4 \bar{B} \nu^{2}\right)\left(r-\frac{V^{\prime}(r)}{V(r)} r^{2}\right)+(G P-E R) r \frac{V^{\prime}(r)}{V(r)}=0, \\
\left(2 J \mp 4 \bar{C} \nu^{2}\right)\left(r-\frac{V^{\prime}(r)}{V(r)} r^{2}\right)+(E Q-F P) r \frac{V^{\prime}(r)}{V(r)}=0,
\end{gathered}
$$


исключение в последних трех уравнениях случаев функции $v(x, y, z)$ вида (I.2)-(I.5) дает условия $H= \pm 2 \bar{A} \nu^{2}, I= \pm 2 \bar{B} \nu^{2}, J= \pm 2 \bar{C} \nu^{2}$ (при этом условие $P H+I Q+$ $R J=0$ оказывается выполненным автоматически) и $F R-G Q=G P-E R=E Q-$ $F P=0$. Переписав последнее условие в виде $E=\theta P, F=\theta Q, G=\theta R$, мы получаем

$$
\begin{aligned}
\xi(x, y, z, \psi)= & \Delta(\psi)\left(P\left(x^{2}-y^{2}-z^{2} \mp \nu^{2}\right)+2 Q x y+2 R x z\right) \\
& +\bar{A}\left(x^{2}-y^{2}-z^{2} \pm \nu^{2}\right)+2 \bar{B} x y+2 \bar{C} x z+\theta(R y-Q z), \\
\eta(x, y, z, \psi)= & \Delta(\psi)\left(Q\left(y^{2}-x^{2}-z^{2} \mp \nu^{2}\right)+2 P x y+2 R y z\right) \\
& +\bar{B}\left(y^{2}-x^{2}-z^{2} \pm \nu^{2}\right)+2 \bar{A} x y+2 \bar{C} y z+\theta(-R x+P z), \\
\zeta(x, y, z, \psi)= & \Delta(\psi)\left(R\left(z^{2}-y^{2}-x^{2} \mp \nu^{2}\right)+2 Q y z+2 P x z\right) \\
& +\bar{C}\left(z^{2}-y^{2}-x^{2} \pm \nu^{2}\right)+2 \bar{B} y z+2 \bar{A} x z+\theta(Q x-P y),
\end{aligned}
$$

что дает алгебру Ли

$$
\begin{aligned}
\Xi_{\mathrm{II} .4}= & \Xi_{\infty}\left(A, B, C, \mp \nu^{2}\right)+\theta \Xi_{\circ}(P, Q, R) \\
& +\left(-\Delta^{\prime}(\psi) S\left(\frac{P x+Q y+R z}{x^{2}+y^{2}+z^{2} \pm \nu^{2}}\right)+\widehat{\varphi}(\psi)\right) \partial_{\psi}
\end{aligned}
$$

с произвольньми константами $\theta, \bar{A}, \bar{B}, \bar{C}$, удовлетворяющими условию $\bar{A} P+\bar{B} Q+$ $\bar{C} R=0$ и с функциями $S(r), V(r), \Delta(\psi)$ и $\widehat{\varphi}(\psi)$, удовлетворяющими уравнению (44), которое является характеристическим уравнением для четвертого семейства.

Здесь, как и ранее, либо $\Delta(\psi) \equiv 0$ и тогда мы приходим к алгебре, обслуживающей все семейство и приведенной в четвертой строке таблицы 2 , либо мы получаем уравнение ветвления

$$
\frac{V^{\prime}(r)}{V(r)}\left(1 \mp 4 r^{2} \nu^{2}\right) \pm 4 r \nu^{2}=\lambda S(r)+\mu
$$

и сопровождаюшие уравнения

$$
\Delta^{\prime \prime}(\psi)+\lambda \Delta(\psi)=0, \quad \hat{\varphi}^{\prime}(\psi)=\mu \Delta(\psi),
$$

которые будут обсуждаться ниже.

6.6. Уравнение ветвления. Как нетрудно видеть, все четыре уравнения ветвления (34), (38), (42), (46) имеют одинаковый вид:

$$
\frac{V^{\prime}(r)}{V(r)} \frac{1}{\chi^{\prime}(r)}-\frac{1}{2}\left(\frac{1}{\chi^{\prime}(r)}\right)^{\prime}=\lambda S(r)+\mu .
$$

В случае первого семейства $\chi^{\prime}(r)=1, \chi(r)=r$, в случае второго $\chi^{\prime}(r)=$ $1 /(4 r), \chi(r)=1 / 4 \ln r$, для третьего $\chi^{\prime}(r)=G^{-1}\left(r^{2}+1+(F+E r)^{2} / G^{2}\right)^{-1}$, $\chi(r)=\operatorname{arctg}\left(\left(r\left(G^{2}+E^{2}\right)+F E\right) / G\right)$ и, наконец, у четвертого семейства $\chi^{\prime}(r)=$ $1 /\left(1 \mp 4 r^{2} \nu^{2}\right)$ и в зависимости от значения $\pm \nu^{2}$ либо

$$
\chi(r)=\int \frac{d r}{1-4 r^{2} \nu^{2}}=\frac{1}{4 \nu} \ln \frac{1+2 \nu r}{1-2 \nu r},
$$


либо

$$
\chi(r)=\int \frac{d r}{1+4 \nu r^{2}}=\frac{1}{2 \nu} \operatorname{arctg} 2 \nu r
$$

либо

$$
\chi(r)=\int d r=r .
$$

Лемма. Пусть $S^{\prime}(r)=1 / V^{2}(r)$. Тогда уравнение (48) имеет следующие решения:

$$
\begin{array}{lll}
V(r)=w \frac{1}{\sqrt{\chi^{\prime}(r)}}, & S(r)=\frac{1}{w^{2}} \chi(r), & \lambda=\mu=0 ; \\
V(r)=w \frac{\exp (k \chi(r))}{\sqrt{\chi^{\prime}(r)}}, & S(r)=-\frac{1}{2 k w^{2}} \exp (-2 k \chi(r)), & \lambda=0, \quad \mu=k ; \\
V(r)=w \frac{\cos (k \chi(r)+h)}{\sqrt{\chi^{\prime}(r)}}, & S(r)=\frac{1}{k w^{2}} \operatorname{tg}(k \chi(r)+h)-\frac{\mu}{\lambda}, & \lambda=-k^{2} w^{2} ; \\
V(r)=w \frac{\operatorname{sh}(k \chi(r)+h)}{\sqrt{\chi^{\prime}(r)}}, & S(r)=-\frac{1}{k w^{2}} \operatorname{cth}(k \chi(r)+h)-\frac{\mu}{\lambda}, & \lambda=-k^{2} w^{2} ; \\
V(r)=w \frac{\operatorname{ch}(k \chi(r)+h)}{\sqrt{\chi^{\prime}(r)}}, & S(r)=-\frac{1}{k w^{2}} \operatorname{th}(k \chi(r)+h)-\frac{\mu}{\lambda}, & \lambda=k^{2} w^{2} ; \\
V(r)=w \frac{\chi(r)+h}{\sqrt{\chi^{\prime}(r)}}, & S(r)=-\frac{1}{w^{2}(\chi(r)+h)}-\frac{\mu}{\lambda}, & \lambda=-w^{2} .
\end{array}
$$

ДоКАЗАТЕЛЬСТво. Если $\lambda=0$, то наше уравнение приобретает вид

$$
\frac{V^{\prime}(r)}{V(r)} \frac{1}{\chi^{\prime}(r)}-\frac{1}{2}\left(\frac{1}{\chi^{\prime}(r)}\right)^{\prime}=\mu
$$

и легко интегрируется:

$$
V^{2}(r)=w \frac{1}{\chi^{\prime}(r)} e^{\mu \chi(r)}
$$

Это - как раз первая (при $\mu=0)$ и вторая (при $\mu \neq 0$ ) из приведенных нами формул, $S(r)$ находится в этом случае простым интегрированием.

Если же $\lambda \neq 0$, то можно заметить, что $\mu$ определяет только выбор $S(r)$ как первообразной от функции $1 / V^{2}(r)$. Поэтому мы можем временно считать $S(r)$ выбранной так, чтобы $\mu$ равнялось нулю (а общий случай получится вычитанием из найденной функции $\mu / \lambda$, как это сделано в формулах с третьей по шестую в формулировке леммы).

Поскольку $S^{\prime}(r)=1 / V^{2}(r)$, а значит, $V^{\prime}(r) / V(r)=-S^{\prime \prime}(r) /\left(2 S^{\prime}(r)\right)$, уравнение (48) приводится к виду

$$
\frac{S^{\prime \prime}(r)}{S^{\prime}(r)} \frac{1}{\chi^{\prime}(r)}+\left(\frac{1}{\chi^{\prime}(r)}\right)^{\prime}=-\lambda S(r) .
$$


После умножения на $S^{\prime}(r)$ оно интегрируется:

$$
S^{\prime}(r) \frac{1}{\chi^{\prime}(r)}=-\lambda\left(S^{2}(r) \pm \varkappa^{2}\right)
$$

Полученное уравнение можно проинтегрировать еще раз, однако интеграл зависит от значения $\pm \varkappa^{2}$ : если оно положительно, то получаем

$$
\begin{gathered}
\frac{1}{\varkappa} \operatorname{arctg} \frac{S(r)}{\varkappa}=-\lambda(\chi(r)+h), \quad S(r)=\varkappa \operatorname{tg}(-\lambda \varkappa(\chi(r)+h)), \\
V(r)=\frac{\cos (-\lambda \varkappa(\chi(r)+h))}{\sqrt{-\lambda \varkappa^{2} \chi^{\prime}(r)}} .
\end{gathered}
$$

Остается переобозначить для удобства $k=-\lambda \varkappa, w=1 /(\sqrt{-\lambda} \varkappa)$, для того чтобы получить третью формулу нашей леммы.

Аналогично, если $\pm \varkappa^{2}$ отрицательно, то (49) дает

$$
\frac{1}{2 \varkappa} \ln \left|\frac{S(r)-\varkappa}{S(r)+\varkappa}\right|=-\lambda(\chi(r)+h), \quad \frac{S(r)-\varkappa}{S(r)+\varkappa}= \pm \exp (-2 \lambda \varkappa(\chi(r)+h))
$$

и в зависимости от того, является \pm плюсом или минусом, получаем либо

$$
S(r)=\varkappa \operatorname{th}(\lambda \varkappa(\chi(r)+h)), \quad V(r)=\frac{\operatorname{ch}(\lambda \varkappa(\chi(r)+h))}{\sqrt{\lambda \varkappa^{2} \chi^{\prime}(r)}},
$$

либо

$$
S(r)=\varkappa \operatorname{cth}(\lambda \varkappa(\chi(r)+h)), \quad V(r)=\frac{\operatorname{sh}(\lambda \varkappa(\chi(r)+h))}{\sqrt{-\lambda \varkappa^{2} \chi^{\prime}(r)}}
$$

Переобозначая, как и выше, $w=1 /(\sqrt{\lambda} \varkappa)$ для первого случая и $w=1 /(\sqrt{-\lambda} \varkappa)$ для второго, $k=-\lambda \varkappa$, мы получим четвертую и пятую формулы нашей леммы.

Наконец, шестая формула получается из (49), если $\varkappa=0$ :

$$
S(r)=\frac{1}{\lambda(\chi(r)+h)}, \quad V(r)=\sqrt{-\lambda} \frac{\chi(r)+h}{\sqrt{\chi^{\prime}(r)}}
$$

$w=\sqrt{-\lambda}$. Доказательство леммы завершено.

Собственно говоря, детальная классификация семейств (II.1)-(II.4) завершена: все формулы таблиц $3-7$ получаются применением леммы для разных функций $\chi(r)$ (и разных значений $r$ ) с подстановкой в алгебры (32), (37), (41), (45) функций, вычисленных при соответствуюших значениях $\lambda, \mu$ из сопровождающих уравнений $(35),(39),(43),(47)$ соответственно. При этом в случае $\lambda \neq 0$ оказывается, что в формулах для $\varphi$ выражения, содержашие $\mu$, просто сокрашаются, так что на конечный результат эта константа не оказывает никакого влияния. Небольшие варианты в сопровождающих уравнениях связаны только с тем, что для разных семейств функция $S(r)$ входит в выражение для $\varphi$ с разными коэффициентами. 
6.7. Случай п. 3.8. Для завершения доказательства нам осталось обсудить случай, который возник в п. 3.8 и обсуждался в п. 5.5 - когда $\xi, \eta$ и $\zeta$ не зависят от $\psi$. В этом случае, как мы уже говорили, $\varphi(x, y, z, \psi)=\widehat{\varphi}(\psi)$, уравнение $(27)$ приобретает вид уравнения с разделенными переменньми, из которого получаем $\widehat{\varphi}^{\prime}(\psi)=M=$ const, $\widehat{\varphi}(\psi)=M \psi+L$ и

$$
\frac{1}{v}\left(\xi v_{x}+\eta v_{y}+\zeta v_{z}\right)=\xi_{x}-M .
$$

Это - линейное неоднородное уравнение в частных производных относительно функции $\omega=2 \ln v(x, y, z)$. По существу, остается только решить это уравнение в тех или иных предположениях. В целях экономии места мы не приводим здесь полные вькладки (они изложены в [16]), а лишь изложим результаты.

В случае $A^{2}+B^{2}+C^{2} \neq 0$ удается сдвигом системы координат привести векторное поле $(\xi, \eta, \zeta)$ к виду

$$
\begin{aligned}
\xi & =\Delta\left(\bar{A}\left(x^{2}-y^{2}-z^{2} \mp \nu^{2}\right)+2 \bar{B} x y+2 \bar{C} x z+\theta(\bar{C} y-\bar{B} z)+\lambda \bar{H}\right), \\
\eta & =\Delta\left(\bar{B}\left(y^{2}-x^{2}-z^{2} \mp \nu^{2}\right)+2 \bar{A} x y+2 \bar{C} y z+\theta(-\bar{C} x+\bar{A} z)+\lambda \bar{I}\right), \\
\zeta & =\Delta\left(\bar{C}\left(z^{2}-x^{2}-y^{2}\right)+2 \bar{A} x z+2 \bar{B} y z+\theta(\bar{B} x-\bar{A} y)+\lambda \bar{J}\right),
\end{aligned}
$$

где $\bar{A}^{2}+\bar{B}^{2}+\bar{C}^{2}=1, \bar{A} \bar{H}+\bar{B} \bar{I}+\bar{C} \bar{J}=0, \bar{H}^{2}+\bar{I}^{2}+\bar{J}^{2}=1$. Введение новой (ортогональной) системы координат $l=\bar{A} x+\bar{B} y+\bar{C} z, s=\bar{H} x+\bar{I} y+\bar{J} z$, $p=(\bar{B} \bar{J}-\bar{C} \bar{I}) x+(\bar{C} \bar{H}-\bar{A} \bar{J}) y+(\bar{A} \bar{I}-\bar{B} \bar{H}) z$ приводит уравнение (50) к виду

$$
\Delta \frac{1}{v}\left(\left(l^{2}-s^{2}-p^{2} \mp \nu^{2}\right) v_{l}+(2 l s+\theta p+\lambda) v_{l}+(2 l p-\theta s) v_{p}\right)=2 \Delta l-M
$$

или, если обозначить $\bar{M}=M / \Delta$,

$$
\frac{1}{v}\left(\left(l^{2}-s^{2}-p^{2} \mp \nu^{2}\right) v_{l}+(2 l s+\theta p+\lambda) v_{l}+(2 l p-\theta s) v_{p}\right)=2 l-\bar{M} .
$$

При этом $\Delta$ оказьвается еще одной (кроме $L$ ) произвольной константой, а интегрирование уравнения (51) в разных случаях (зависящих от соотношений между коэффициентами $\lambda, \theta, \pm \nu^{2}$, определяемых в терминах корней биквадратного уравнения $\left.\left(\mu^{2}+\theta^{2}\right)\left(\mu^{2} \mp 4 \nu^{2}\right)-4 \lambda^{2}=0\right)$ дает семейства функций, приведенные в таблице 8 , которьм отвечает алгебра

$$
\Xi=\Delta\left(\left(l^{2}-s^{2}-p^{2} \mp \nu^{2}\right) \partial_{l}+(2 l s+\theta p+\lambda) \partial_{s}+(2 l p-\theta s) \partial_{p}+M \psi \partial_{\psi}\right)+L \partial_{\psi} .
$$

$\mathrm{K}$ сожалению, уравнение (51) поддается интегрированию лишш отчасти: первьй, рациональный интеграл этого уравнения находится всегда, а второй, трансцендентньй - только в частных случаях. Это - единственное место во всей работе, где результат не удалось довести до явных формул.

В случае же $A=B=C=0$ векторное поле $(\xi, \eta, \zeta)$ оказьвается линейньп и уравнение $(50)$ интегрируется явным образом. В зависимости от того, равны или нет нулю $E^{2}+F^{2}+G^{2}, D, H^{2}+I^{2}+J^{2}$, получаются варианты, приведенные в таблице 9.

В случае же, когда $\xi=\eta=\zeta \equiv 0$, мы получаем из уравнения (50) $M=0$, и это дает нам алгебру Ли $\Xi=L \partial_{\psi}$, обслуживающую все уравнения эйконала, которая, впрочем, для данного уравнения является тривиальной: она порождает группу сдвигов по переменной $\psi$, а то, что сдвиг $\psi$ не меняет уравнения, - очевидно.

Таким образом, доказательство нашей теоремы полностью завершено. 
§ 7. Геометрия фронтов и лучей для уравнения с функциями (I.2)-(I.5)

7.1. Фронты волн точечного источника. Будемговорить, что поверхность $t=\psi(x, y, z)$ в пространстве $\mathbb{R}^{3}$ описывает фронт волнь точечного источника, если $\psi(x, y, z)$ является решением уравнения $(1)$ и $\psi^{-1}(0)$ состоит из единственной точки $\left(x_{0}, y_{0}, z_{0}\right)$ (которую мы и называем источником).

Фронт волны от точечного источника для постоянной функции скорости $v(x, y, z) \equiv v=$ const известен - это сфера радиуса $v t$ :

$$
\left(x-x_{0}\right)^{2}+\left(y-y_{0}\right)^{2}+\left(z-z_{0}\right)^{2}=v^{2} t^{2} .
$$

Оказывается, сферическим является фронт не только для постоянной, но и для остальных главных функций скорости (Т.2)-(Т.5).

Teорема 2. Пусть $v(x, y, z)=P x+Q y+R z\left(\right.$ әде $\left.P^{2}+Q^{2}+R^{2}=w^{2}>0\right)$. Тогда фронт волны точечного источника, находящегося в точке $\left(x_{0}, y_{0}, z_{0}\right)$, является сферическим и определяется соотношением

$$
\left(x-x_{0}-P \rho(t)\right)^{2}+\left(y-y_{0}-Q \rho(t)\right)^{2}+\left(z-z_{0}-R \rho(t)\right)^{2}=r^{2}(t),
$$

в котором

$$
\rho(t)=\frac{P x_{0}+Q y_{0}+R z_{0}}{w^{2}}(\operatorname{ch} w t-1), \quad r(t)=\frac{P x_{0}+Q y_{0}+R z_{0}}{w} \operatorname{sh} w t .
$$

В случае $(P, Q, R)=(w, 0,0)$ (скорость меняется вдоль оси $x)$ формула (52) приобретает вид $\left(x-x_{0} \operatorname{ch} w t\right)^{2}+\left(y-y_{0}\right)^{2}+\left(z-z_{0}\right)^{2}=\left(x_{0} \operatorname{sh} w t\right)^{2}$.

При $t \rightarrow+\infty$ сфера, расширяясь, приближается к "плоскости горизонта" $P x+$ $Q y+R z=0$.

Из (52) следует, в частности, что центр сферы (псевдоисточник), находящийся в точке $\left(x^{*}, y^{*}, z^{*}\right)=\left(x_{0}, y_{0}, z_{0}\right)+(P, Q, R) \rho(t)$ на расстоянии $r=r(t)$ от точки $(x, y, z)$ фронта волны, движется относительно нее со скоростью, равной $\dot{\rho} \cdot(P, Q, R)=r \cdot \nabla v\left(x^{*}, y^{*}, z^{*}\right)$, т.е. равной произведению расстояния от этой точки до псевдоисточника и градиента функции $v(x, y, z)$.

Tеорема 3. Пусть $v(x, y, z)=w \cdot\left(x^{2}+y^{2}+z^{2} \pm \nu^{2}\right)$. Тогда фронт волны от точечного источника, расположсенного в точке $\left(x_{0}, y_{0}, z_{0}\right)$, является сферическим и определяется соотношением

$$
\left(x-x_{0} \rho(t)\right)^{2}+\left(y-y_{0} \rho(t)\right)^{2}+\left(z-z_{0} \rho(t)\right)^{2}=r^{2}(t),
$$

где функиии $\rho(t)$ и $r(t)$ задаются формулами (всюду $\left.r_{0}^{2}=x_{0}^{2}+y_{0}^{2}+z_{0}^{2}\right)$ :

$$
\begin{gathered}
\rho(t)=\frac{\nu^{2}\left(1+\operatorname{tg}^{2} w \nu t\right)}{\nu^{2}-r_{0}^{2} \operatorname{tg}^{2} w \nu t}, \quad r(t)=\frac{\nu\left(r_{0}^{2}+\nu^{2}\right) \operatorname{tg} w \nu t}{\nu^{2}-r_{0}^{2} \operatorname{tg}^{2} w \nu t}, \\
v(x, y, z)=w \cdot\left(x^{2}+y^{2}+z^{2}+\nu^{2}\right), \\
\rho(t)=\frac{\nu^{2}\left(1-\operatorname{th}^{2} w \nu t\right)}{\nu^{2}-r_{0}^{2} \operatorname{th}^{2} w \nu t}, \quad r(t)=\frac{\nu\left(r_{0}^{2}-\nu^{2}\right) \operatorname{th} w \nu t}{\nu^{2}-r_{0}^{2} \operatorname{th}^{2} w \nu t}, \\
v(x, y, z)=w \cdot\left(x^{2}+y^{2}+z^{2}-\nu^{2}\right), \\
\rho(t)=\frac{1}{1-r_{0}^{2} w^{2} t^{2}}, \quad r(t)=\frac{r_{0}^{2} w t}{1-r_{0}^{2} w^{2} t^{2}}, \\
v(x, y, z)=w \cdot\left(x^{2}+y^{2}+z^{2}\right) .
\end{gathered}
$$


При обрашении в (54)-(56) знаменателя в нуль сфера вырождается в плоскость $x x_{0}+y y_{0}+z z_{0}=\left(r_{0}^{2} \mp \nu^{2}\right) / 2$.

$\mathrm{B}$ первом случае при $\operatorname{tg} w \nu t=\infty$ сфера стягивается в инверсную к $\left(x_{0}, y_{0}, z_{0}\right)$ точку $-\nu^{2}\left(x_{0}, y_{0}, z_{0}\right) / r_{0}^{2}$, а при $\operatorname{tg} w \nu t=0$ - в исходную точку $\left(x_{0}, y_{0}, z_{0}\right)$. Во втором - при $t \rightarrow \infty$ и при $r_{0}>\nu$ она стремится к "сфере горизонта" $x^{2}+y^{2}+z^{2}=\nu^{2}$ снаружи; при $r_{0}<\nu$ сфера в плоскость никогда не вырождается, а, расширяясь, стремится при $t \rightarrow \infty$ к той же “сфере горизонта" $x^{2}+y^{2}+z^{2}=\nu^{2}$, но изнутри. В третьем - при $t \rightarrow \infty$ сфера стягивается к началу координат.

Из формулы (53) следует, что центр сферы (псевдоисточник), находящийся в точке $\left(x^{*}, y^{*}, z^{*}\right)=\left(x_{0}, y_{0}, z_{0}\right) \rho(t)$ на расстоянии $r=r(t)$ от точки $(x, y, z)$ фронта волны, двигается относительно нее со скоростью, равной $2 w r\left(x^{*}, y^{*}, z^{*}\right)=$ $r \nabla v\left(x^{*}, y^{*}, z^{*}\right)$, т.е. равной произведению расстояния от точки $(x, y, z)$ до псевдоисточника и градиента в точке псевдоисточника.

Отметим, что формулы (52), (53) (если в них вместо $t$ подставить $\psi$ ) являются полными интегралами Лагранжа (с тремя параметрами $\left.x_{0}, y_{0}, z_{0}\right)$ уравнения (1), и поэтому любое решение может быть найдено как огибающая соответствуюшего семейства сфер при условии задания некоторой связи $F\left(x_{0}, y_{0}, z_{0}\right)=0$ (поверхности начального условия).

ДоКАЗАТЕЛЬСТво ТЕОРЕм 2,3 осуществляется подстановкой вместо $t$ функции $\psi(x, y, z)$ и проверкой того, что так определенная функция удовлетворяет уравнению (I.2) или уравнениям (I.3)-(I.5) соответственно. Наличие анзаца делает результат легко вычисляемым, однако получить этот анзац было существенно сложнее, и, сказать откровенно, он для автора оказался неожиданным открытием.

\section{2. Геометрия лучей.}

Tеорема 4. Пусть $v(x, y, z)=P x+Q y+R z\left(P^{2}+Q^{2}+R^{2}>0\right)$. Тогда лучи (семейство кривых, ортогональных фронтам) являются полуокружностями, лежсащими в плоскостях, ортогональных плоскости горизонта $P x+Q y+R z=0$, и опирающимися на плоскость горизонта.

Тем самым в среде с линейной функцией скорости лучи порождают в точности геометрию Лобачевского (соответствующей модели Пуанкаре в полуплоскости).

ДоКАЗАТЕЛЬСТво сводится к повороту системы координат так, чтобы оказалось $v(x, y, z)=w x$, и проверке того, что семейство лучей, описываемых формулами

$$
x=x_{0} \frac{\operatorname{ch} C}{\operatorname{ch}(w t+C)}, \quad y=y_{0}+x_{0} \frac{\cos \theta \operatorname{sh} w t}{\operatorname{ch}(w t+C)}, \quad z=z_{0}+x_{0} \frac{\sin \theta \operatorname{sh} w t}{\operatorname{ch}(w t+C)}
$$

при подстановке в (42) дает тождество и что это семейство ортогонально семейству сфиер (42). Углы $\varphi=\arccos \operatorname{cth} C$ и $\theta-$ полярные углы касательной к лучу в момент $t=0$.

Tеорема 5. Пусть $v(x, y, z)=w \cdot\left(x^{2}+y^{2}+z^{2}-\nu^{2}\right)($ әде $\nu>0)$. Тогда лучи являются дугами окружностей, лежсащих в плоскостях, проходящих через начало координат, и эти дуги опираются на сферу горизонта $x^{2}+y^{2}+z^{2}=\nu^{2}$. 
Отметим, что сфера $x^{2}+y^{2}+z^{2}=\nu^{2}$ разделяет пространство на две несвязанные части, в каждой из которых волновой процесс происходит независимо от другой (внутри сферы предполагается, что $w<0$, а вне сферы - что $w>0$ ). Таким образом, для функции скорости (І.3) лучи порождают геометрию Лобачевского либо внутри сферы (что соответствует модели Пуанкаре в круге), либо вне сферы - в инвертированном относительно этой сферы варианте.

Tеорема 6. Пусть $v(x, y, z)=w \cdot\left(x^{2}+y^{2}+z^{2}+\nu^{2}\right)$ (где $\left.\nu>0\right)$. Тогда лучи являются окружностями, проходящими через исходную точку $\left(x_{0}, y_{0}, z_{0}\right)$ и инверсную ей $-\nu^{2}\left(x_{0}, y_{0}, z_{0}\right) /\left(x_{0}^{2}+y_{0}^{2}+z_{0}^{2}\right)$.

Таким образом, для функции скорости (I.4) лучи порождают сферическую геометрию.

Tеорема 7. Пусть $v(x, y, z)=w \cdot\left(x^{2}+y^{2}+z^{2}\right)$. Тогда лучи являются окружностями, проходящими через исходную точку $\left(x_{0}, y_{0}, z_{0}\right)$ и начало координат.

В этом случае геометрия лучей оказывается уже обычной евклидовой; преобразование в привычную нам геометрию осуществляется при помощи инверсии $r \mapsto$ $-r /\|r\|^{2}$, устанавливающей эквивалентность уравнений эйконала для функций $v(x, y, z)$ вида (I.1) и (I.5).

ДОКАЗАТЕЛЬСТВо ТЕОРЕМ $5-7$ состоит в ортогональном преобразовании системы координат так, чтобы оказалось $x_{0}=r_{0}, y_{0}=0, z_{0}=0$, и в проверке того, что семейство окружностей, задаваемых формулами

$$
x=\widehat{x}+R \frac{1-u^{2}}{1+u^{2}}, \quad y=\left(-C+R \frac{2 u}{1+u^{2}}\right) \cos \theta, \quad z=\left(-C+R \frac{2 u}{1+u^{2}}\right) \sin \theta
$$

где

$$
\widehat{x}=\frac{1}{2}\left(x_{0} \mp \frac{\nu^{2}}{x_{0}}\right), \quad R^{2}=C^{2}+\left(\widehat{x}-x_{0}\right)^{2}, \quad u=\frac{C+r(t)}{R-\widehat{x}+x_{0} \rho(t)},
$$

при подстановке в (53) дает тождество и что это семейство ортогонально семейству сфер (53): касательный вектор

$$
(\dot{x}, \dot{y}, \dot{z})=2 R \frac{\dot{u}}{1+u^{2}}\left(\frac{2 u}{1+u^{2}}, \frac{1-u^{2}}{1+u^{2}} \cos \theta, \frac{1-u^{2}}{1+u^{2}} \sin \theta\right)
$$

пропорционален вектору нормали к поверхности фронта

$$
\begin{gathered}
\nabla \psi=\frac{1}{v(x, y, z) r}\left(x-x_{0} \rho(t), y, z\right)=\frac{1}{v(x, y, z) r}\left(\widehat{x}-x_{0} \rho(t)+R \frac{1-u^{2}}{1+u^{2}},\right. \\
\left.\left(-C+R \frac{2 u}{1+u^{2}}\right) \cos \theta,\left(-C+R \frac{2 u}{1+u^{2}}\right) \sin \theta\right) .
\end{gathered}
$$

Существенно упрощает доказательство тождество $\left(\widehat{x}-x_{0} \rho(t)\right)^{2}=\left(\widehat{x}-x_{0}\right)^{2}+r^{2}(t)$, которое проверяется непосредственно по формулам (54)-(56). 


\section{Список литературы}

1. Курант Р. Уравнения с частными производньгии. М.: Мир, 1964.

2. Бреховских Л. М. Волны в слоистых средах. М.: Наука, 1973.

3. Бреховских Л. М., Годин О. А. Акустика слоистых сред. М.: Наука, 1989.

4. Бабич B. М., Булдырев В. С. Асимптотические методы в задачах дифракции коротких волн. М.: Наука, 1972.

5. Соболев С. Л. Волновое уравнение для неоднородной среды // Труды Сейсмологического ин-та. 1930. №6. С. 1-57.

6. Кравцов Ю.А., Орлов Ю.И. Геометрическая оптика неоднородных сред. М.: Наука, 1980.

7. Бабич В.М., Булдырев В. С., Молотков И. А. Пространственно-временной лучевой метод: линейные и нелинейные волны. Л.: Из-во ЛГУ, 1985.

8. Арнольд В. И. Геометрия сферических кривых и алгебра кватернионов // УМН. 1995. Т. 50. № 1. С. 3-68.

9. Арнольд В. И. Топологические проблемы теории распространения волн // УМН. 1996. T. 51. № 1. C. 3-50.

10. Смирнов В.И. Курс высшей математики. Т. IV. М.: ГТТЛ, 1953.

11. Итоги науки и техники. Современные проблемы математики. Т. 22. М.: ВИНИТИ, 1983.

12. Овсянников Л. В. Групповой анализ дифференциалњных уравнений. М.: Наука, 1978.

13. Ибрагимов Н. Х. Групшы преобразований в математической физике. М.: Наука, 1983.

14. Олвер П. Приложения групп Ли к дифференциалњным уравнениям. М.: Мир, 1989.

15. Ибрагимов H. Х. Группы Ли в некоторых вопросах математической физики. Новосибирск: Из-во НГУ, 1972.

16. Боровских A. В. Групповая классификация уравнений эйконала для волнового уравнения в неоднородной среде // Деп. в ВИНИТИ 29.05.2002 № 953-В2002.

Воронежский государственный университет

E-mail: bor.bor@mail.ru

Поступила в редакцию

06.11 .2002 